\title{
Activity-dependent calpain activation plays a critical role in synaptic facilitation and post-tetanic potentiation
}

\author{
Arkady Khoutorsky and Micha E. Spira ${ }^{1}$ \\ Department of Neurobiology, The Life Sciences Institute, The Hebrew University of Jerusalem, Jerusalem 91904, Israel
}

\begin{abstract}
Synaptic facilitation and post-tetanic potentiation (PTP) are believed to necessitate active regeneration of the release machinery and supply of synaptic vesicles to a ready-releasable site. The prevailing hypothesis assumes that synapsins play pivotal roles in these processes. Using a cholinergic synapse formed between cultured Aplysia neurons (B2 and MCn), we demonstrate here that the calcium-activated protease-calpain serves as a major regulating element in the cascade that links electrical activity, elevation of the free intracellular calcium concentration, and short-term synaptic enhancements such as facilitation and PTP. Our study revealed that calpain inhibitors (calpeptin and MG132) transform a facilitating synapse into a depressing one, and reduce its PTP by $80.6 \%$. Inhibition of CaM kinases, PKA, and MAPK also reduced PTP at this synapse. When inhibitors of these kinases were applied together with calpeptin, tetanic stimuli led to synaptic depression. We concluded that at this synapse facilitation and PTP are mediated mainly by the calpain-dependent processes and to a smaller extent by the CaMKs/PKA/MAPK-dependent cascades.
\end{abstract}

The strength of chemical synapses can be modulated for short or long periods of time. At excitatory synapses, depletion of the readily releasable vesicle stores by high-frequency stimulation has been recognized as a major cause of short-term synaptic depression (Model et al. 1975; Rosenmund and Stevens 1996; Weis et al. 1999; Wu and Borst 1999). Inactivation of presynaptic $\mathrm{Ca}^{2+}$ currents and other downstream mechanisms that lower the release probability were also proposed to account for homosynaptic depression (Patil et al. 1998; Wu and Borst 1999; Royer et al. 2000; Gover et al. 2002; Xu et al. 2007). Short-term synaptic enhancement, on the other hand, is attributed to a number of activity-dependent mechanisms including enhanced calcium (Ca) influx (Jackson et al. 1991; Geiger and Jonas 2000; Habets and Borst 2005), reduced Ca buffering (Blatow et al. 2003; Felmy et al. 2003), increased channel-vesicle coupling, or increased Ca sensitivity of the vesicle fusion mechanisms (Korogod et al. 2007). In addition, activity-dependent recruitment of synaptic vesicles to the release sites is implied to play a critical role in short-term enhancement of release (Stevens and Wesseling 1998; Wang and Kaczmarek 1998). The recruitment of vesicles is manifested either by accelerated transport of vesicles from a feeding pool, which stores vesicles, to a ready-releasable pool (Gingrich and Byrne 1985; Wang and Kaczmarek 1998; Zhao and Klein 2004; Fioravante et al. 2007; Habets and Borst 2007; Neher and Sakaba 2008) and/or by efficient recycling of exocytosed vesicles (Ceccarelli et al. 1973; Heuser and Reese 1973; Sudhof 2004; Schweizer and Ryan 2006). A large number of studies postulate that accelerated supply of vesicles from the feeding pool to the release sites is initiated by activity-dependent elevation of the free intracellular Ca concentration $\left(\left[\mathrm{Ca}^{2+}\right]_{\mathrm{i}}\right)$ at the synaptic terminal (Gingrich and Byrne 1985; Dittman and Regehr 1998; Stevens and Wesseling 1998; Wang and Kaczmarek 1998; Hosoi et al. 2007). The mechanisms by which the elevated $\left[\mathrm{Ca}^{2+}\right]_{\mathrm{i}}$ is translated to vesicle recruitment have been the subject of intense studies. These revealed that the

\footnotetext{
'Corresponding author.
}

E-mail spira@cc.huji.ac.il; fax 972-2-5637033.

Article is online at http://www.learnmem.org/cgi/doi/10.1101/lm.1275709. synaptic protein family of synapsins plays a key role in the process. Synapsins that bind to form homo- and hetero-multimers also bind to presynaptic actin meshwork and tether synaptic vesicles, thereby forming a pool of synaptic vesicles that are not free to translocate within the presynaptic terminal-the reserve vesicle pool (Greengard et al. 1993; Brodin et al. 1997; Hilfiker et al. 1999; Fdez and Hilfiker 2006). Dissociation or tethering of vesicles is regulated by synapsin phosphorylation and dephosphorylation, respectively. Synapsin isoforms are phosphorylated by cAMP-dependent protein kinase (PKA), mitogen-activated protein kinase (MAPK), and $\mathrm{Ca}^{2+} /$ calmodulin-dependent protein kinases (CaMK) I, II, and IV (Fdez and Hilfiker 2006). Consequently, synapsin phosphorylation or dephosphorylation is thought to modulate synaptic facilitation and depression (Nakanishi et al. 1997; Jovanovic et al. 2000; Fioravante et al. 2007; Fiumara et al. 2007). Post-docking role of synapsin in synaptic vesicles exocytosis has also been suggested (Rosahl et al. 1993; Hilfiker et al. 1998; Humeau et al. 2001). The central role of synapsin in the regulation of synaptic release was challenged by observations suggesting that the mobility of synaptic vesicles and release dynamics was unchanged in the neuromuscular junction of the synapsin triple knock-out mouse (Gaffield and Betz 2007).

Whereas synapsin-based regulation of vesicle pools is at the center of interest, a growing number of reports suggest that the family of Ca-activated neutral proteases, calpains may also be involved in regulating synaptic transmission in an activitydependent manner. Currently most reports emphasize the regulatory function of calpain at the postsynaptic level (Wu and Lynch 2006), yet a small number of studies provided experimental evidence that calpain activation also regulates rapid neurotransmitter release processes (Khoutorsky and Spira 2005) and secretion of neurohormones (Evans and Turner 2007).

In an earlier study, we used Aplysia sensory-motoneuron synapse (Byrne and Kandel 1996) to study the role of calpain in 5HT-induced facilitation of depressed synapse. This synapse exhibits homosynaptic depression when stimulated at low frequencies. A single bath application of serotonin (5HT) leads, within minutes, to facilitation of the depressed synapse. The 
facilitation was attributed to mobilization of neurotransmitter containing vesicles from a "feeding vesicle pool" (Pieribone et al. 1995), also referred to as a "reserve pool" (Zucker and Regehr 2002; Fioravante et al. 2007), to a depleted readily releasable pool (Rosenmund and Stevens 1996; Schikorski and Stevens 2001). We previously reported that the calpain inhibitors calpeptin, MG132, and ALLN enhance the rate of homosynaptic depression and block the 5HT-induced facilitation of depressed synapses (Khoutorsky and Spira 2005). The data suggested that two processes are involved in replenishing the depleted ready-releasable vesicle stores: (1) 5HT activates Ca-independent PKC (Manseau et al. 2001), which stimulates the translocation of the vesicles or changes their functional status, and (2) Ca activates calpain, which proteolyses an unknown barrier that impedes the translocation of vesicles from the feeding pool to a ready-releasable pool.

In the present study, we examined whether calpain operates in processes that enable synaptic release under stimulation frequencies that lead to enhanced release (in the forms of facilitation and post-tetanic potentiation [PTP]). And if so, what is the relative contribution of calpain in respect to the PKA/CaMKs/MAPK pathways vis-à-vis facilitation and PTP? The experiments reported here were conducted on a cholinergic synapse formed between buccal neuron 2 (B2) and the metacerebral neuron (MCn) in culture. This synapse is characterized by low quantal content and significant facilitation when stimulated at $>1 \mathrm{~Hz}$ and reveals PTP. In the presence of calpain inhibitors, the synapse is transformed into a depressing one and PTP is impaired. PKA, CaMKs, and MAPK inhibitors reduce the PTP, but only MAPK cascade inhibition alters synaptic facilitation. Our results indicate that the elevation of the $\left[\mathrm{Ca}^{2+}\right]_{i}$ by tetanic stimulation activates calpain and phosphorylates synapsin by PKA/CaMKs/MAPK activation. We suggest that both processes contribute to PTP of the B2-MCn synapse. Since the concomitant inhibition of calpain and any of the synapsin phosphorylating kinases (PKA/CaMKs/MAPK) led to a post-tetanic depression (PTD) rather than PTP, we suggest that regeneration of the release machinery and possibly vesicles replenishment are completely arrested when both pathways are blocked. Under these conditions tetanic stimulus causes failure of the release machinery or/and exhaustion of the ready-releasable pool of presynaptic vesicles and PTD.

\section{Results}

\section{Characterization of the synaptic system used in the present study}

Cultured Aplysia neurons have been extensively used to study the biophysical and molecular mechanisms of short- and long-term synaptic enhancement as they allow long-term recording and stimulation from the pre- and postsynaptic neurons (Bailey and Kandel 2008). The majority of the experiments were conducted on synapses formed between the sensory and motoneurons of the gill withdrawal reflex (Bailey and Kandel 2008). This synapse undergoes depression when stimulated at very low frequencies. For the purpose of the present study we were searching for a synapse that reveals synaptic facilitation and PTP rather than undergoes depression (habituation). To that end we screened a number of neuronal pairs and found that B2 and the MCn form a chemical synapse with the appropriate properties. Although these neurons do not form synapses in vivo, $87 \%$ of the cocultured pairs form chemical synapses $(n>100)$. In culture, the neurites of B2 form varicosities on the poly-L-lysine-coated glass substrate (Malkinson et al. 2006) and in contact with the main MCn's axon (Fig. 1). The mean number of varicosities formed by $\mathrm{B} 2$ on the main MCn's axon is $4.7 \pm 1.4$ (counted on the third day of the culture, $n=8$ pairs).
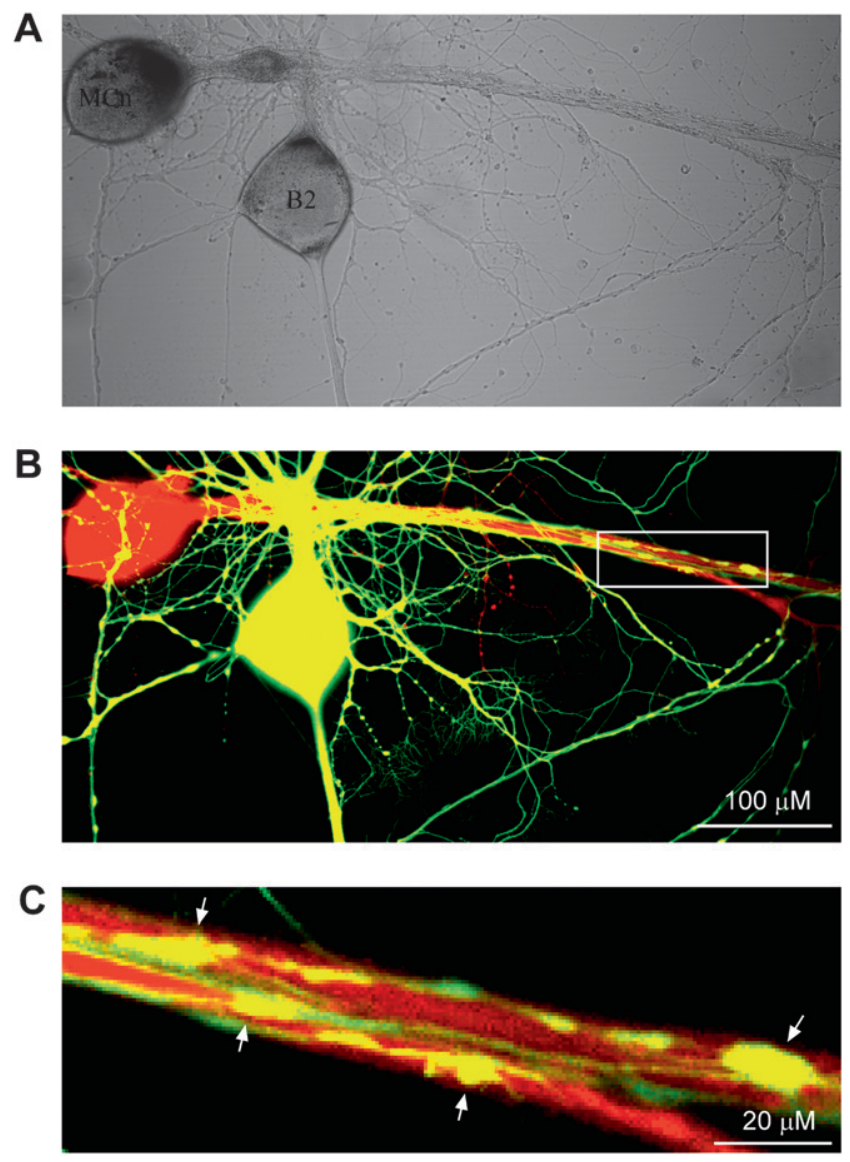

Figure 1. Buccal neuron 2 (B2) forms en passant varicosities in contact with the axon of metacerebral neurons (Mcn) in culture. (A) Transmitted light micrograph of cocultured B2 and MCn $3 \mathrm{~d}$ after plating. $(B)$ The same neurons stained by intracellular injection of carboxifluorescein (B2, green) and Alexa Fluor 555 hydrazide (MCn, red). Note the extensive growth of the presynaptic neuron on the main shaft of the MCn. (C) B2 varicosities are formed in contact with the MCn (inset in $B$ ).

Intracellular stimulation of B2 evokes postsynaptic potentials (PSPs) recorded from the MCn cell body as early as $6 \mathrm{~h}$ after the neurons were plated $(n=4)$. Synaptic potentials could be evoked for $>10 \mathrm{~d}$ in culture $(n=5)$. The reversal potential of the synapse is above the threshold to fire action potentials, $-31.6 \pm 3.24 \mathrm{mV}$ (Fig. 2A,B; $n=4$ ), and trains of stimuli delivered to the presynaptic neuron generate summating PSPs that reach threshold to fire action potentials (Fig. 2C,D). Thus, the synapse is defined as an excitatory synapse.

The resting potential of cultured postsynaptic neurons ranged between 45 and $54 \mathrm{mV}$. The amplitude of PSPs recorded from MCns at a transmembrane potential of $-60 \mathrm{mV}$ ranged from 0.54 to $5.6 \mathrm{mV}$, averaging $1.4 \pm 0.19 \mathrm{mV}$ on the third day after plating $(n=19)$. Spontaneous miniature excitatory postsynaptic potentials (mEPSPs) were recorded infrequently. In a few experiments in which mEPSPs were recorded $(7 />100)$, the mean mEPSPs frequency was $0.03 / \mathrm{sec}$, with mean amplitude of $0.29 \pm 0.01 \mathrm{mV}$ $(n=27)$. Calculation of the quantal content in these experiments (average evoked EPSPs amplitude/average mEPSP amplitude) yielded a mean quantal content value of $6.25 \pm 1.04$. This value agrees well with the quantal content calculated by the coefficient of variation method (Del Castillo and Katz 1954; Castellucci and Kandel 1974), which gave a mean quantal content of $7.495 \pm 1.59$ $(n=5)$. 
A

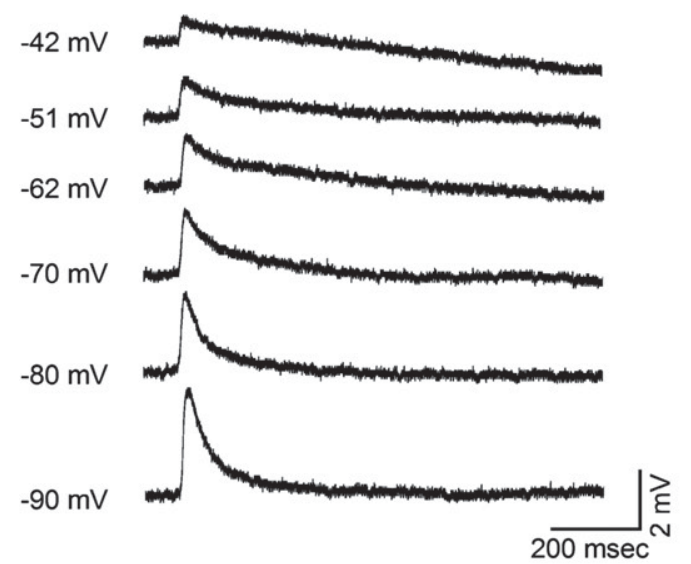

C

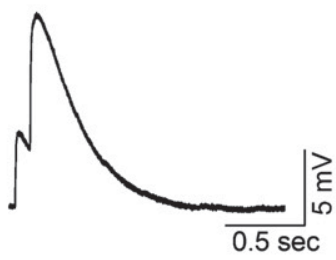

$\mathbf{E}$

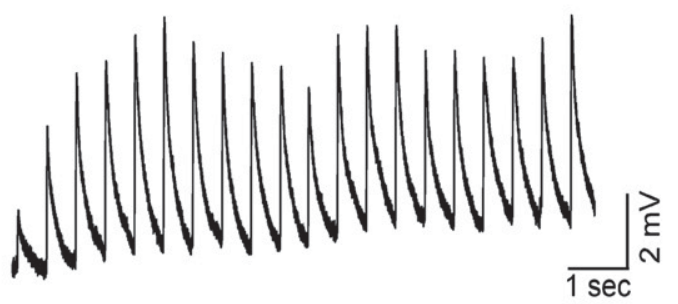

B

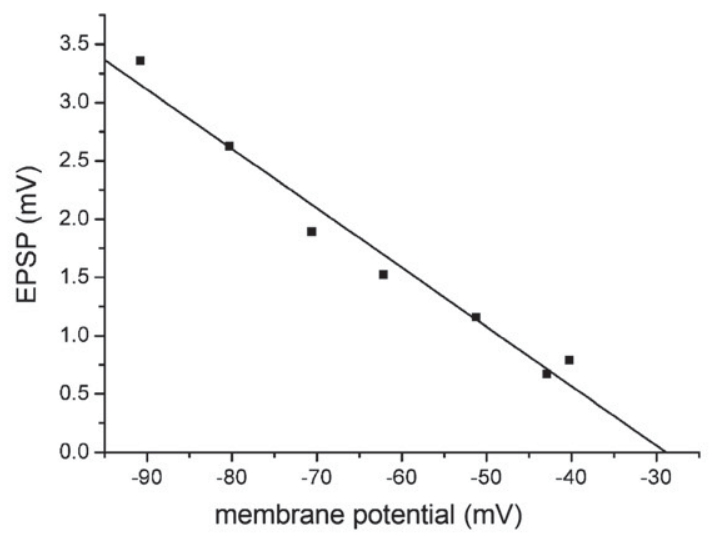

D
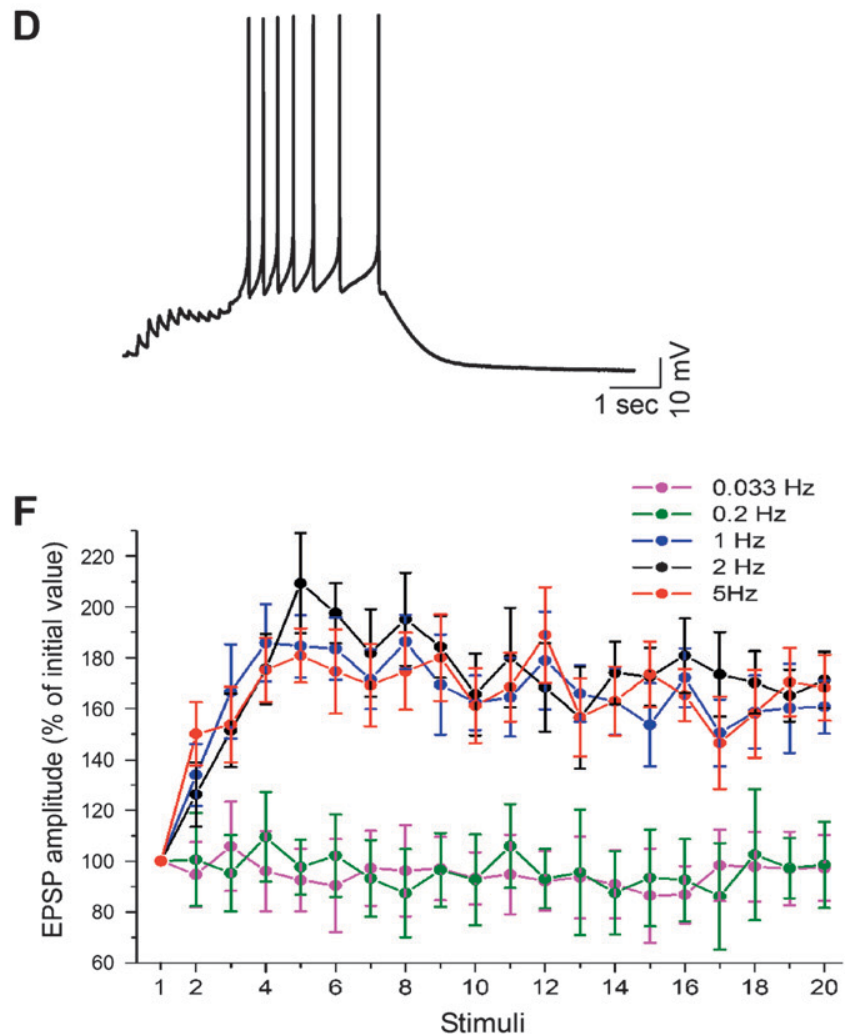

Figure 2. Characterization of the B2-MCn synapse. The reversal potential of the synapse was estimated by plotting the postsynaptic potential amplitude generated by presynaptic stimulation in relation to the transmembrane potential of the postsynaptic neuron. The postsynaptic membrane potential was set to different values (ranging from -90 to $-40 \mathrm{mV}$ ) by current injections. $(A)$ EPSPs recorded at different postsynaptic transmembrane potentials (indicated on the left-hand side of the traces). (B) The relation between the PSP amplitude and the transmembrane potential of the postsynaptic neuron. The extrapolated value of the reversal potential of the synapse is $-31.6 \pm 3.24 \mathrm{mV}(n=4)$. The synapse demonstrates paired-pulse facilitation $(C$, interpulse interval $100 \mathrm{msec}$ ), summates to fire action potentials $(D$, stimulation frequency $5 \mathrm{~Hz})$, and facilitates $(E, F)$. ( $E)$ Sample recording showing EPSP facilitation upon stimulation at $2 \mathrm{~Hz}$. ( $P$ ) B2-MCn synapse facilitates when stimulated at $5 \mathrm{~Hz}(n=5), 2 \mathrm{~Hz}(n=6)$, and $1 \mathrm{~Hz}(n=5)$ but not when stimulated at $0.2 \mathrm{~Hz}(n=5)$ or $0.033 \mathrm{~Hz}(n=5)$.

To pharmacologically define the synapse, we screened a number of classical inhibitors. We found that $100 \mu \mathrm{M}$ d-tubocurarine reduced the mean EPSP amplitude to $19.33 \pm 6.3 \%$ of the initial value ( $n=5$, not shown) and $100 \mu \mathrm{M}$ hexamethonium reduced it to $25.39 \pm 5.2 \%$ of the initial value ( $n=3$, not shown). The synaptic potentials were totally blocked by the muscarinic antagonist atropine at $0.5 \mathrm{mM}(n=4$, not shown). Thus, by pharma- cological criteria, the synapse can be defined as a cholinergic synapse (Kehoe 1972).

The B2-MCn synapse presents paired-pulse facilitation (Fig. 2C) and readily facilitates, upon stimulation, at frequencies $>1 \mathrm{~Hz}$ (Fig. 2E,F), reaching a plateau $\sim 1.6$ times larger than the control EPSP. At frequencies $<1 \mathrm{~Hz}$, the mean EPSP amplitude was maintained rather constant. 


\section{The effect of calpain inhibition on the synaptic properties}

To test the hypothesis that activity-dependent calpain activation plays a role in facilitation and PTP, we inhibited calpain activation by the membrane-permeable inhibitor, calpeptin (Tsujinaka et al. 1988; Gitler and Spira 1998, 2002; Khoutorsky and Spira 2005). We found that (1) preincubation of the neurons in $100 \mu \mathrm{M}$ calpeptin for $15 \mathrm{~min}$ led to increased amplitude of the first EPSP from $1.7 \pm 0.4 \mathrm{mV}$ in the control to $4.5 \pm 1 \mathrm{mV}$ in the presence of calpeptin (Fig. 3D; $n=6, P<0.05$; paired $t$-test); (2) in contrast to the control experiments, which revealed synaptic facilitation, in the presence of calpeptin, the EPSP amplitude was rapidly depressed (Fig. 3A,B; $F_{(49,490)}=2.943, P<0.001$; repeated measures one-way ANOVA, $n=6$ ). The depression rate was dependent on the stimulus frequency (Fig. 3C). At a stimulation frequency of $2 \mathrm{~Hz}$, the PSP was depressed to a steady-state level of $\sim 15 \%$ of the control, with a time constant of $2 \mathrm{sec}$. At a stimulation rate of 0.2 $\mathrm{Hz}$, the time constant for depression was slower, $-31 \mathrm{sec}$, and the extent to which the synapse was depressed was $\sim 59 \%$ of the initial value. When the synapse was stimulated at a slower rate of once every $3 \mathrm{~min}(0.0055 \mathrm{~Hz})$, the depression was very mild and the synapse strength was reduced to $\sim 80 \%$ of the initial value (Fig. $3 C$ ).

The increase in the first PSP amplitude is attributed to the increase in the spike duration of the presynaptic neuron due to decreased voltage-gated potassium conductances as recently described by us (Khoutorsky and Spira 2008). In the B2 neurons, calpain application increases the spike duration from $2.67 \pm 0.39$ msec in control to $32 \pm 7 \mathrm{msec}$ in calpeptin $(n=7$, measured from the peak of the action potential to when the membrane potential reached 33\% of the peak, $P<0.01$, independent samples $t$-test).

The transformation of this homosynaptic facilitating synapse into a depressing one by calpeptin could be attributed to a number of mechanisms: (1) Spike broadening, which leads to increased Ca influx and increased release of vesicles, could enhance the rate of vesicles depletion from the ready-releasable vesicle stores; (2) the calpain inhibitor calpeptin could progressively reduce the $\mathrm{Ca}$ influx into presynaptic terminals or enhance the rate of Ca removal from the cytosole. Inhibition of calpain could theoretically also reduce the Ca sensitivity of the vesicle fusion mechanism; (3)
A

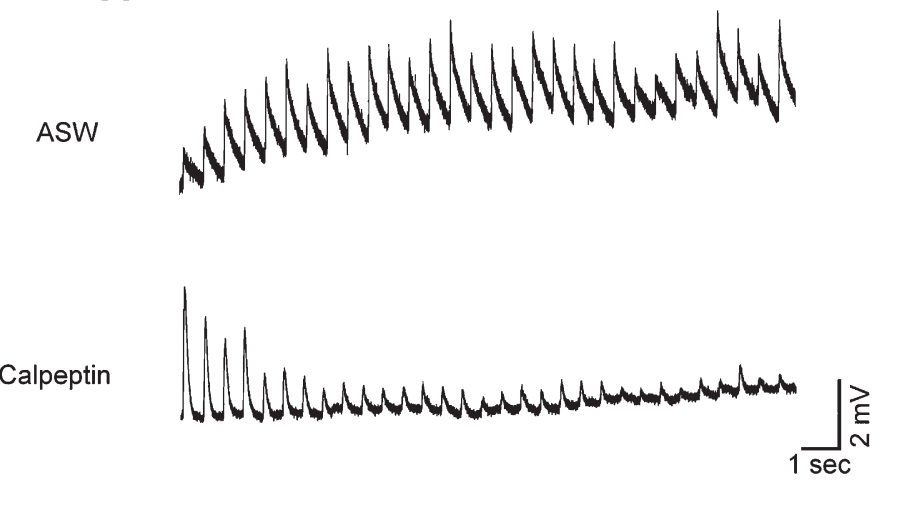

C



B
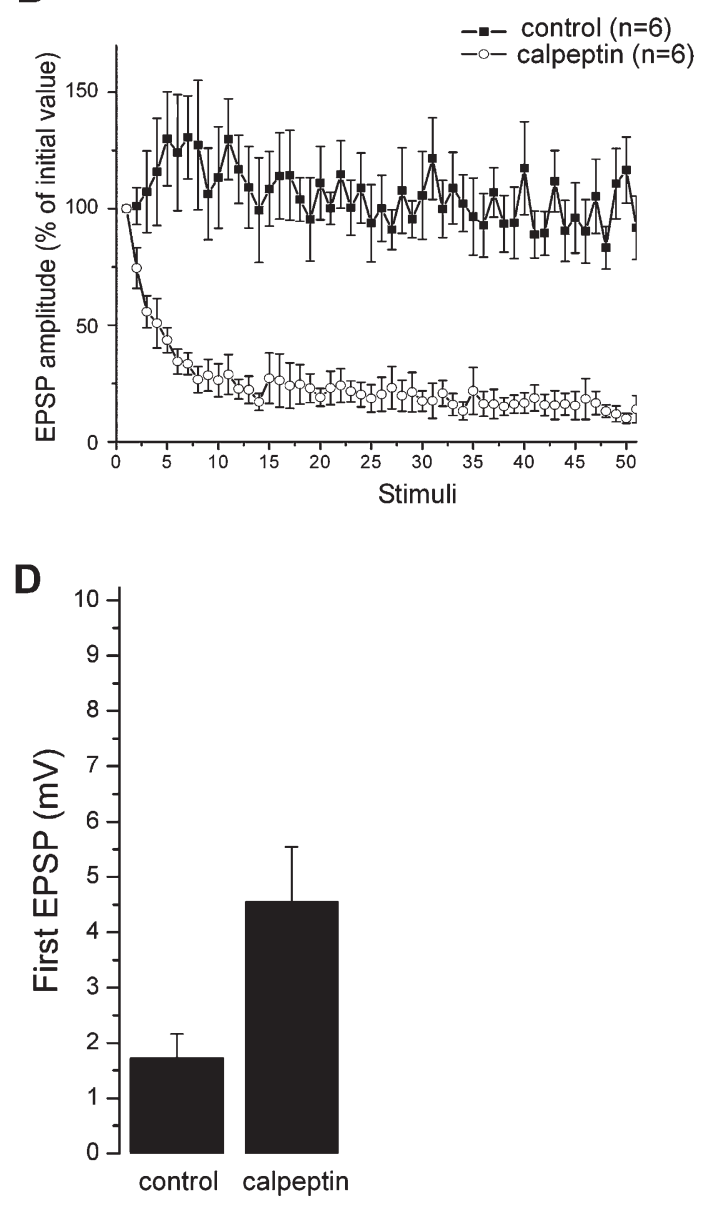

Figure 3. Transformation of a facilitating synapse into a depressing one by the calpain-inhibitor calpeptin. ( $A$ ) Evoked EPSPs generated by stimulation of $\mathrm{B} 2$ at $2 \mathrm{~Hz}$ and recorded from a MCn's cell body in ASW (control) and after 15 min of incubation in $100 \mu \mathrm{M}$ calpeptin. Note that the application of calpeptin leads to increased PSP amplitude $(A, D)$ and that, whereas in ASW the synapse facilitates, in the presence of calpeptin the EPSP's amplitude is depressed $(A, B)$. (B) The mean values of normalized EPSP amplitudes in control experiments $(n=6)$ and in the presence of calpeptin $(n=6)$, as a function of stimuli delivered at a rate of $2 \mathrm{~Hz}$. The rate of the EPSPs' amplitude depression in the presence of calpeptin increases with increased stimulus frequency. (C) The final level reached at different frequencies is inversely related to the frequency. (D) The mean amplitude of the first EPSP recorded in ASW (control, $1.72 \pm 0.43 \mathrm{mV}, n=6)$ is significantly smaller than the mean amplitude of the first EPSP in calpeptin (calpeptin, $4.54 \pm 1, n=6, P<0.01 ;$ paired $t$-test). 
calpeptin could lead to blockade of the postsynaptic receptors; and (4) calpain inhibition could reduce the activity-dependent rate of recovery of the release machinery, including recruitment of vesicles to the release site by vesicle mobilization or by membrane recycling.

To test the first hypothesis, we performed two types of experiments. First we increased evoked release by increasing the Ca concentration of the bathing solution. To that end the normal artificial sea water (ASW) (containing $11 \mathrm{mM} \mathrm{Ca}$ and $55 \mathrm{mM} \mathrm{Mg}$ ) was replaced by a low-magnesium $(11 \mathrm{mM})$ high-Ca $(55 \mathrm{mM})$ solution. Under these conditions, the mean amplitude of the first EPSP was increased from $1.7 \pm 0.4$ to $7.4 \pm 1.7 \mathrm{mV}$ (Fig. $4 \mathrm{~A} ; n=6$, $P<0.01$; independent samples $t$-test), the initial phase of synaptic facilitation was not detected, and the EPSP amplitude was gradually depressed when repeatedly stimulated. The rate of depression was significantly slower than that following the inhibition of calpain (Fig. $4 \mathrm{~B} ; F_{(49,490)}=3.136, P<0.001$; repeated measures oneway ANOVA, $n=6$ ).

In the second set of experiments we examined the consequences of reducing synaptic release by lowering the Ca concentration in the bathing solution to $5 \mathrm{mM}$ and elevating the magnesium to $61 \mathrm{mM}$. Under these conditions the amplitude of the EPSP was reduced to a level that could hardly be reliably detected. Nevertheless, after the addition of $100 \mu \mathrm{M}$ calpeptin the spike was broadened and the mean amplitude of the first EPSP recovered to the level of the first EPSP in normal ASW (Fig. 4A; average first EPSP amplitude $1.17 \pm 0.3 \mathrm{mV}, n=8, P=0.226$; independent samples $t$-test). Under these conditions (in the presence of calpeptin) the depression rate of the EPSPs was significantly faster in respect to the control experiments (Fig. 4B; $F_{(49,245)}=5.15, P<0.001$; repeated measures one-way ANOVA, $n=6$ ).

Since the increased release in high Ca resulted in depression rate that is slower than in the presence of calpeptin, and calpeptin accelerated the rate of depression even under conditions of low release, we conclude that increased release as a result of spike broadening in calpeptin is not the underlying mechanism for transforming the facilitating B2-MCn synapse to a depressing one.

To examine whether calpeptin reduces the influx of $\mathrm{Ca}$ ions into the presynaptic neuron during consecutive firing of action potentials, or facilitates its removal, we loaded the presynaptic neurons with the Ca indicator fluo- 4 and measured the Ca levels within varicosities in contact with the main MCn's axon during presynaptic stimulation (Fig. 5). We found that the generated $\left[\mathrm{Ca}^{2+}\right]_{\mathrm{i}}$ transient profiles in calpeptin are not reduced considerably during repetitive stimulation at $0.1 \mathrm{~Hz}$, while the EPSPs' amplitudes are greatly attenuated. The recovery rate of the $\left[\mathrm{Ca}^{2+}\right]_{i}$ was not enhanced in the presence of calpeptin (mean time constant of $\mathrm{Ca}$ decay $1.21 \pm 0.04 \mathrm{sec}$ in ASW, and $1.16 \pm 0.05 \mathrm{sec}$ in calpeptin, $n=$ $4, P>0.05)$. These observations are inconsistent with the hypothesis that calpeptin reduces $\mathrm{Ca}$ influx or accelerates Ca removal from the presynaptic varicosities. As the spatiotemporal resolution of $\mathrm{Ca}$ imaging does not allow us to measure alterations of the free intracellular $\mathrm{Ca}$ concentrations within the microdomains of the release sites, and as the release sites are distributed among a number of varicosities, we could not evaluate whether the Ca sensitivity of vesicle fusion mechanisms was altered by calpeptin.

To examine the hypothesis that calpeptin operates postsynaptically by blocking the receptors, we pressure-ejected brief ACh $(100 \mu \mathrm{M})$ pulses from a micropipette onto the MCn membrane while monitoring the membrane potential with an intracellular microelectrode (Kehoe and McIntosh 1998). Since ACh-induced depolarization lasted 1-3 sec, the rate of ACh application was reduced to $0.05 \mathrm{~Hz}$. We found that bath application of calpeptin does not reduce the response amplitude to ACh (Fig. 6; $n=4$ ), while the synaptic EPSP amplitude in calpeptin was
A

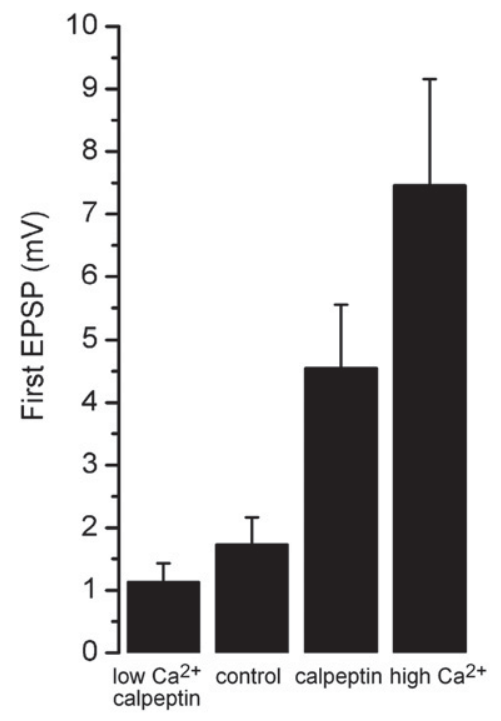

B

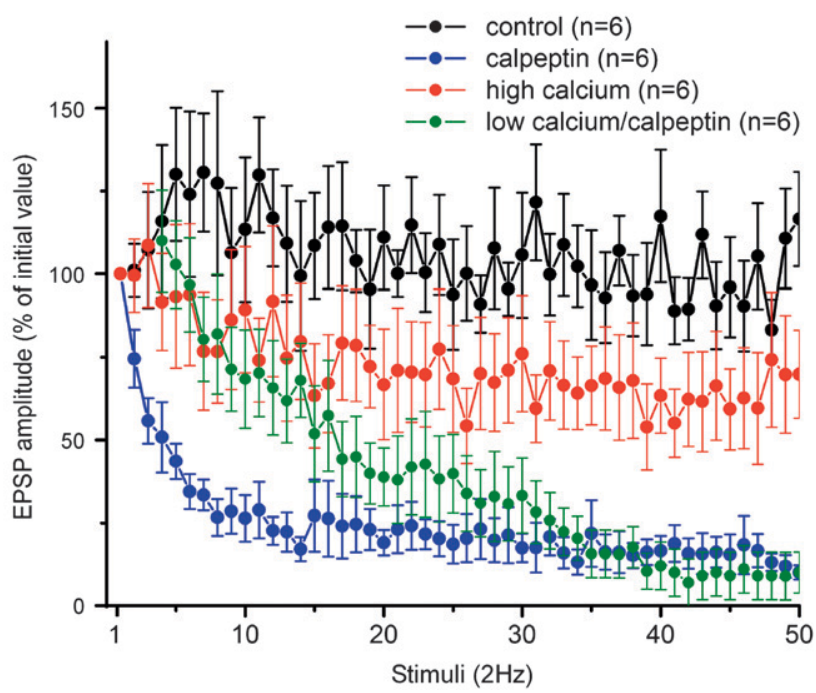

Figure 4. The accelerated depression of the B2-MCn synapse in calpeptin is not caused by increased Ca influx and increased quantal content. To test whether synaptic depression in the presence of calpeptin results from increased neurotransmitter release, we performed two experiments. First, we increased the quantal content of the evoked EPSPs by elevating the extracellular Ca concentration and reducing the magnesium concentration (for details, see text). In a high-Ca low-magnesium solution, the first EPSP was significantly larger than in calpeptin $(A, 7.45 \pm 1.7 \mathrm{mV}$ and $4.54 \pm 1, n=6$, respectively). The depression rate was tested by stimulating the presynaptic neuron at $2 \mathrm{~Hz}$. In the presence of a high-Ca low-magnesium solution, the rate of homosynaptic depression was significantly slower than in the presence of calpeptin $(B, n=6)$. In the second experiment we reduced the extracellular Ca concentration from $11 \mathrm{mM}$ in ASW to $5 \mathrm{mM}$, and elevated the magnesium concentration to $61 \mathrm{mM}$. In the presence of calpeptin the first EPSP was $1.17 \pm 0.3 \mathrm{mV}(A)$. We found that under these conditions of low release the depression rate in calpeptin was significantly faster than in control $(B, n=6)$. 
A

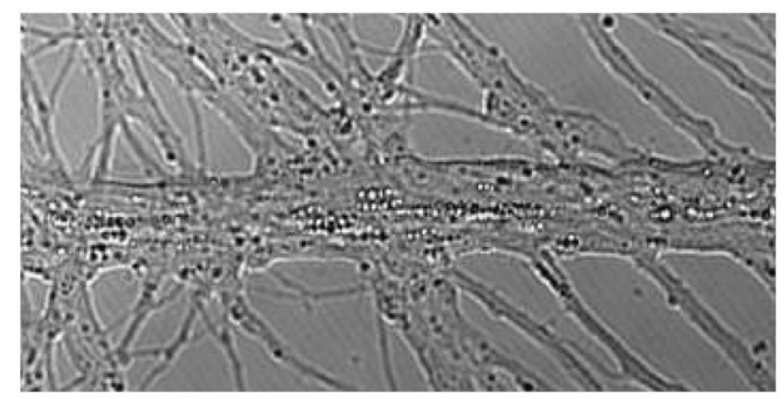

Cl

control

1

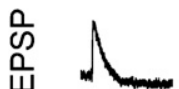

II



III

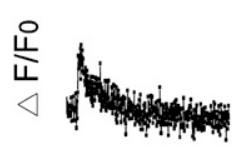

DI



II
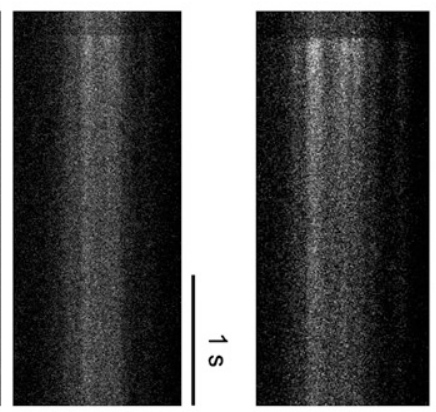

III

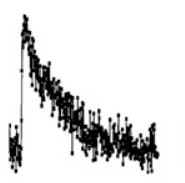

B

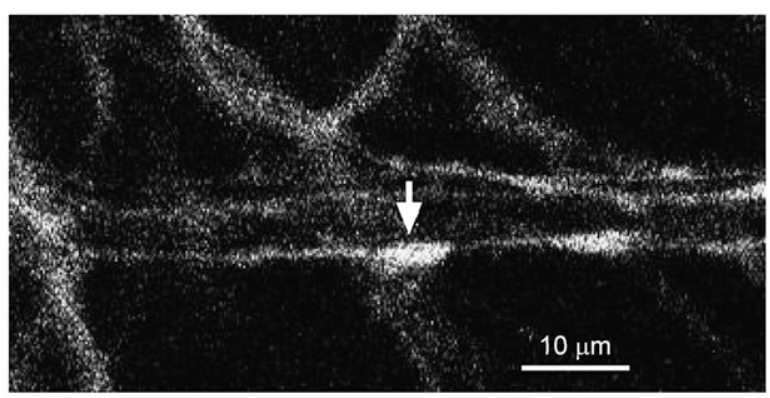

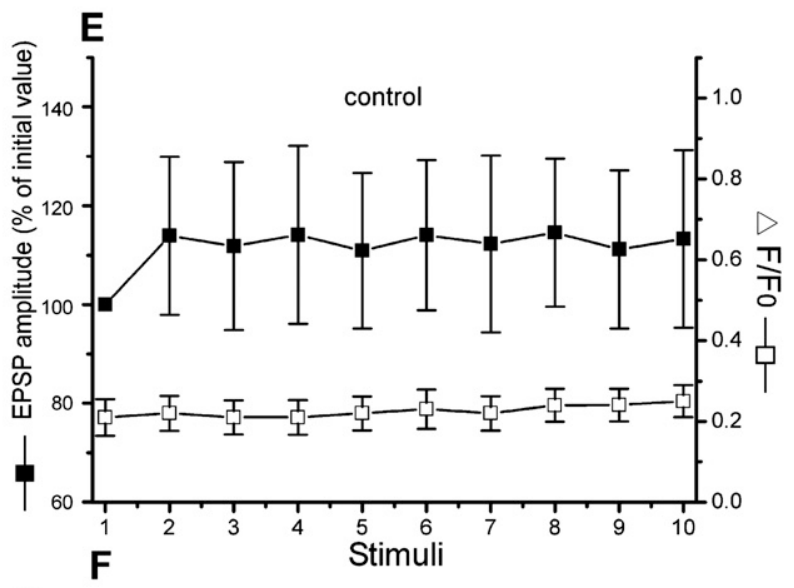

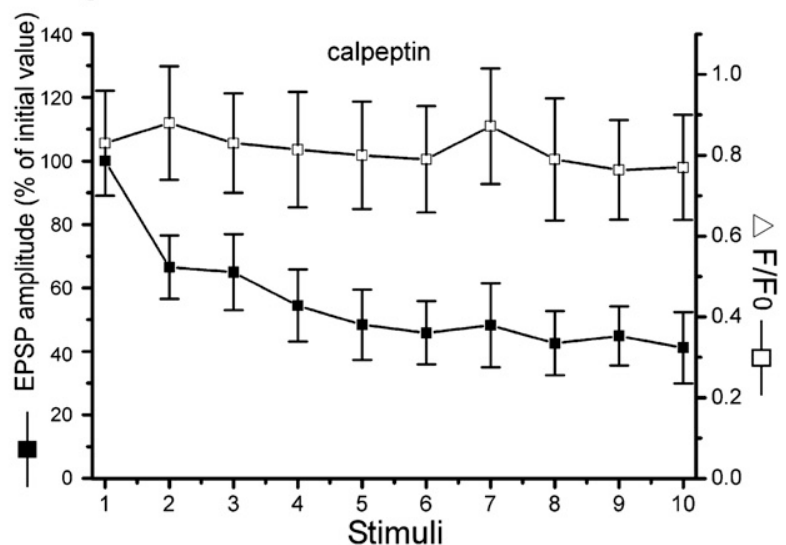

Figure 5. The Ca dynamics in the presynaptic varicosities is not altered by calpeptin. The effect of calpeptin on Ca dynamics in the course of evoked release was imaged in control neurons and after calpeptin application. For the experiments, B2 neurons were microinjected with fluo-4 pentapotassium and the fluorescent intensity of the presynaptic varicosities was monitored by line scans. $(A)$ A transmitted light microscope micrograph of a MCn segment. Under these conditions, the presynaptic varicosities cannot be recognized. (B) Imaging of the fluo-4 fluorescence reveals varicosities in close apposition to the MCn's axon. For the experiment, the presynaptic neuron was stimulated at $0.1 \mathrm{~Hz}$ to fire 10 action potentials. Concomitantly, EPSPs were intracellularly recorded (in control, $\mathrm{Cl}$, and in calpeptin, $\mathrm{DI}$ ), while the fluo-4 fluorescent signal was line-scanned at the location indicated by the arrow in $B$ (CII and DII). The line scans were analyzed off line and the changes in the free Ca concentration are represented as $\Delta \mathrm{F} / \mathrm{FO}$ (CIII and DIII). (D) The experiment was conducted first in ASW (control, $C$ ) and then after 15-min rest in the presence of $100 \mu \mathrm{M}$ calpeptin on the same neuron. The average values of EPSPs (normalized, black squares, $n=3$ ) and of $\Delta$ F/FO fluo-4 (empty squares, $n=3$ ) in control $(E)$ and in calpeptin $(F)$ show that the Ca concentration transients in calpeptin are only slightly reduced in the course of 10 stimuli, whereas the EPSPs' amplitudes are depressed substantially, indicating that depression of the PSP amplitude is not due to decreased Ca transients in the presynaptic terminals.

reduced to $66 \%$ after 30 stimuli under the same stimulation rate (not shown, $n=4$ ).

Based on the above results we next examined the hypothesis that calpain activation by Ca influx is an essential step in the cascade of events that lead to the facilitation of synaptic release. To test this hypothesis, we first examined whether another calpain inhibitor exerts effects similar to those observed with calpeptin. We then analyzed the effects of calpain inhibition on post-tetanic potentiation.

We found that, whereas $100 \mu \mathrm{M}$ MG132, a calpain and proteasome inhibitor (Figueiredo-Pereira et al. 1994; Lee and Goldberg 1998), transformed the release kinetics from facilitation to depression (Fig. 7A; $F_{(49,490)}=1.8, P<0.005$; repeated measures 


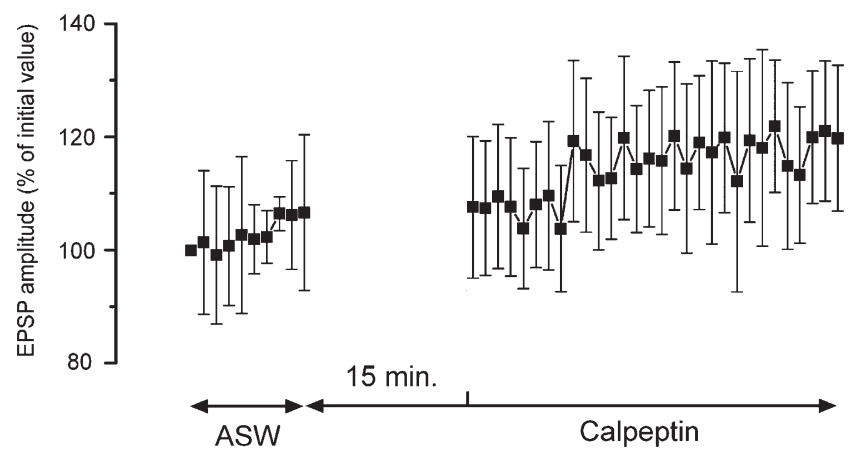

Figure 6. The transient depolarization of a MCn to local ACh application is not affected by calpeptin. To examine the possible postsynaptic effect of calpeptin, we monitored the transmembrane potential of $\mathrm{MCn}$ while pressure-ejecting $100 \mu \mathrm{M} \mathrm{ACh}$ onto the initial segment of the $\mathrm{MCn}$ before and after applying $100 \mu \mathrm{M}$ calpeptin to the bathing solution. After $10 \mathrm{ACh}$ ejection pulses at $0.05 \mathrm{~Hz}$ in ASW, calpeptin was applied and 15 min later the ACh applications were resumed $(n=4)$.

one-way ANOVA, $n=6$ ), the specific membrane-permeable proteasome inhibitor, clasto-lactacystine $\beta$-lactone $10 \mu \mathrm{M}$ (Fenteany et al. 1995; Dick et al. 1996), had no effect on the release kinetics $\left(F_{(49,392)}=1.327, P=0.08\right.$; repeated measures one-way ANOVA, $n=$ 4; Fig. 7A). It is thus concluded that the facilitating synapse is transformed into a depressing one by calpain inhibitors.

\section{Post-tetanic potentiation and calpain inhibition}

The effect of calpain inhibition on PTP was examined in the following manner: To establish the pre-tetanic EPSP amplitude, we evoked five EPSPs by intracellular stimulation of the presynaptic neuron at a low frequency of $0.033 \mathrm{~Hz}$. Thereafter, a tetanic train of stimuli $(20 \mathrm{~Hz})$ was delivered for $2 \mathrm{sec}$, leading to summation of the EPSPs (Fig. 8A). Thirty seconds after the tetanic stimulation was terminated, the pre-tetanic stimulation rate was resumed. In control experiments, the peak amplitude of PTP was 306\% $\pm 27 \%$ (Fig. $8 \mathrm{~B} ; n=16$ ) of the mean pre-tetanic value. To examine the effects of spike-evoked increased $\mathrm{Ca}$ influx into the presynaptic terminal on PTP, we replaced the normal ASW (containing $11 \mathrm{mM}$ $\mathrm{Ca}^{2+}$ and $\left.55 \mathrm{mM} \mathrm{Mg}^{2+}\right)$ by a high-Ca ${ }^{2+}(55 \mathrm{mM})$ low- $\mathrm{Mg}^{2+}(11 \mathrm{mM})$ solution. This led, as mentioned earlier, to an increase in the first EPSP's amplitude by a factor of 4.35 (Fig. 4A). Under these conditions, the mean peak amplitude of the PTP was 390\% \pm $57 \%$. This value does not differ significantly from the PTP value obtained in the control experiments $(n=5, P>0.05$, independent samples $t$-test). The effect of calpeptin on PTP was studied following $15 \mathrm{~min}$ of preincubation in $100 \mu \mathrm{M}$ calpeptin. Under these conditions, the peak amplitude of the PTP was reduced to $140 \% \pm 25 \%$ of the pre-tetanic value, and significantly differed from the control PTP (Fig. $8 ; n=6, P<0.01$, independent samples $t$ test). To test whether increased release in calpeptin is the reason for decreased PTP, we measured the PTP in low-Ca high-magnesium (5 and $61 \mathrm{mM}$, respectively) calpeptin $(100 \mu \mathrm{M})$ solution. As indicated earlier the mean first EPSP recorded in this solution was $1.17 \pm 0.3 \mathrm{mV}$ (Fig. 4A). We found that in calpeptin and low $\mathrm{Ca}$ the PTP was significantly reduced (Fig. $8 \mathrm{~B} ; n=3, P<0.01$, independent samples $t$-test), indicating that the PTP is blocked in calpeptin even under conditions of low transmitter release.

\section{The relative contribution of CaM kinases, PKA, and MAPK and calpain-dependent processes to post-tetanic potentiation}

The observation that, in the presence of calpeptin, partial PTP is still observed suggested that additional mechanisms contribute to
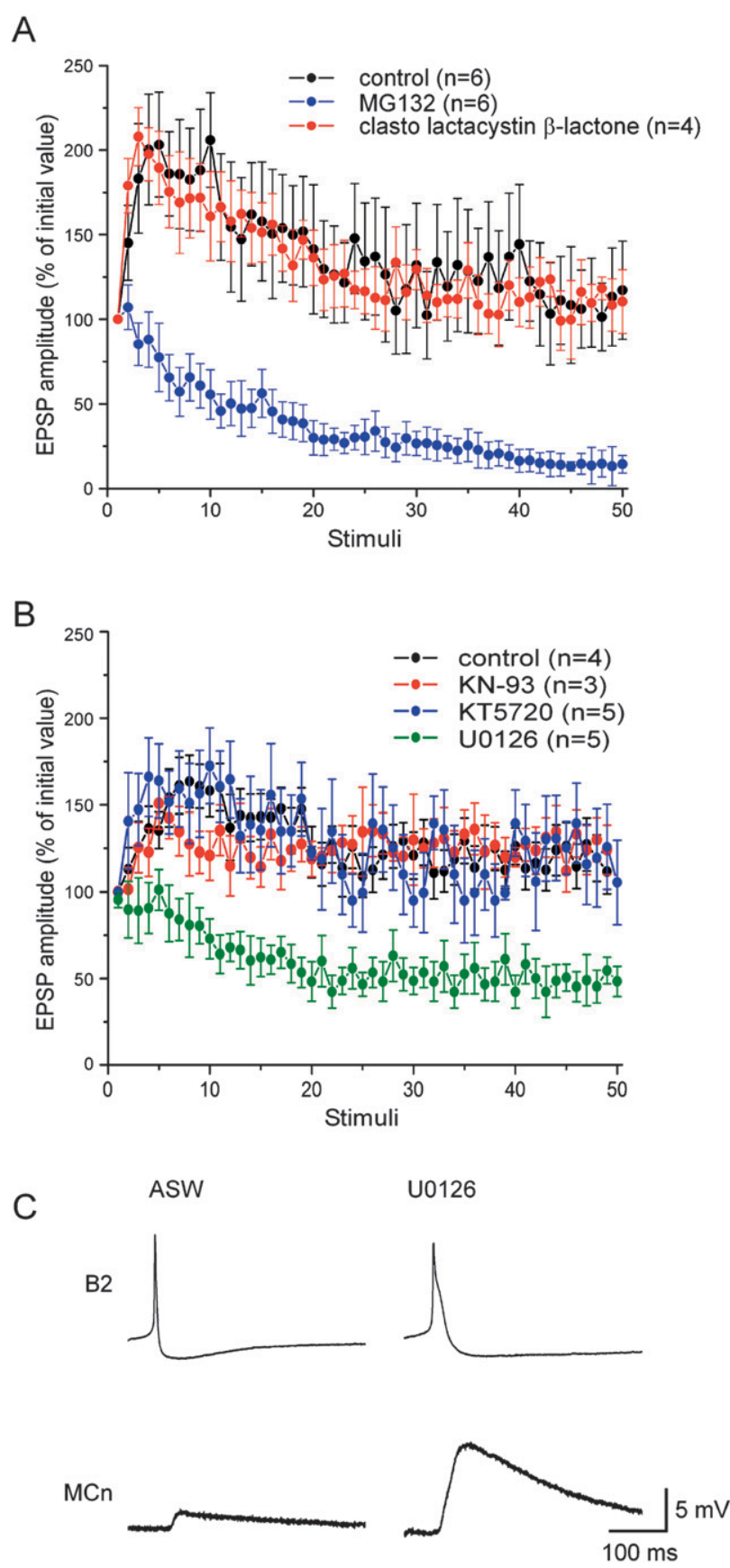

Figure 7. The effects of MG132, clasto-lactacystine $\beta$-lactone, and protein kinases inhibitors on the release kinetics of the B2-MCn synapse. The effects of MG132, clasto-lactacystine $\beta$-lactone, KN-93, KT5720, and $\mathrm{U} 0126$ on the kinetics of synaptic release were studied in a similar manner to that described for calpeptin in the experiments of Figure 3 by delivering intracellular stimulation to the presynaptic neuron at a stimulation frequency of $2 \mathrm{~Hz}$. (A) Whereas $100 \mu \mathrm{M}$ MG132 (blue) significantly accelerated synaptic depression ( $n=6$ for control and MG132), $10 \mu \mathrm{M}$ of clasto-lactacystine $\beta$-lactone (red) had no effect on synaptic transmission $(n=4)$. (B) Likewise, incubation of the synapse for $15 \mathrm{~min}$ in $10 \mu \mathrm{M} \mathrm{KN}-93$ (red) or $10 \mu \mathrm{M} \mathrm{KT5720} \mathrm{(blue)} \mathrm{had} \mathrm{no} \mathrm{significant} \mathrm{effect} \mathrm{on} \mathrm{transmitter}$ release when stimulated at a frequency of $2 \mathrm{~Hz}$ (for KN-93, $n=3$, and for KT5720, $n=5$ ). When the synapse was incubated for 15 min in MEK inhibitor U0126 (B, green, $20 \mu \mathrm{M})$, the action potential, recorded in the B2 neuron, was prolonged and the EPSP amplitude was increased ( $C$, see text for details). Stimulation of $B 2$ neuron at $2 \mathrm{~Hz}$ in the presence of U0126 resulted in increased rate of depression ( $B$, green, $n=5)$. 
A
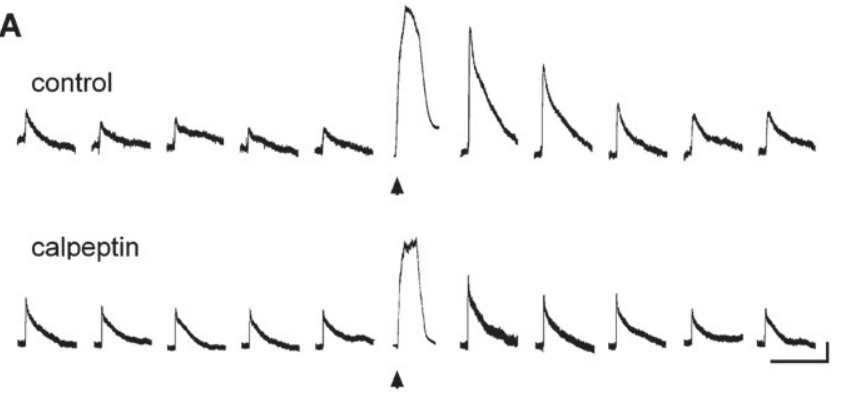

B

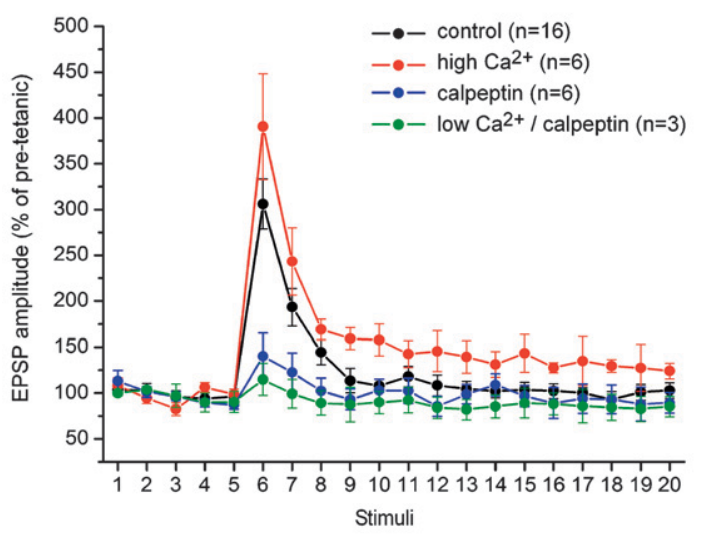

C

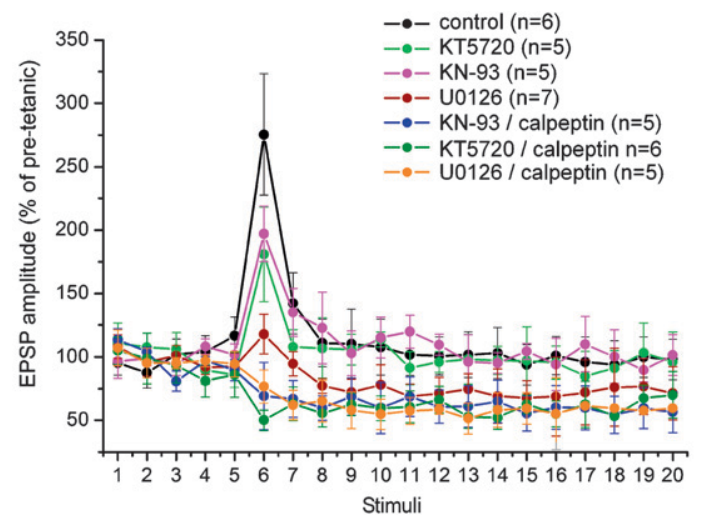

Figure 8. Inhibitors of calpain, MAPK, PKA, and CaM kinases impair PTP (A) Sample electrophysiological PTP recording of the B2-MCn synapse. Action potentials were generated by intracellular stimulation of the presynaptic B2 neuron at $0.033 \mathrm{~Hz}$, while recording EPSPs in MCn. After five control stimuli, a tetanic stimulus (20 Hz for $2 \mathrm{sec}$, black arrow) was delivered. Thirty seconds later, the pre-tetanic stimulation frequency was resumed. The post-tetanic EPSPs' amplitude increased significantly $30 \mathrm{sec}$ after the tetanus and progressively declined during the next 2-3 min. (B) Summary of PTP experiments conducted in normal ASW-control experiments (black), in a high-Ca low-magnesium solution (red, $55 \mathrm{mM} \mathrm{Ca}$ and $11 \mathrm{mM}$ magnesium), in calpeptin dissolved in normal ASW (blue, $15 \mathrm{~min}$ in $100 \mu \mathrm{M})$, and in a low-Ca-calpeptin solution (green, $5 \mathrm{mM} \mathrm{Ca}$ and $61 \mathrm{mM}$ magnesium). Note that in the presence of calpeptin in ASW or low Ca the PTP was greatly reduced. (C) The effects of 15-min incubation of MEK inhibitor U0126 (purple, $20 \mu \mathrm{M}$ ), PKA inhibitor KT5720 (light green, 10 $\mu \mathrm{M}$ ), and $\mathrm{CaM}$ kinases inhibitor $\mathrm{KN}-93$ (blue, $10 \mu \mathrm{M}$ ) on PTP were investigated. We found that U0126, KT5720, and KN-93 significantly decreased the PTP. When U0126, KT5720, or KN-93 was applied together with calpeptin, the tetanic stimulation was followed by synaptic depressions. Values are normalized with respect to the average amplitude of the five pre-tetanic EPSPs. Calibration $(A)$ for the EPSPs $1 \mathrm{mV}$ and $1 \mathrm{sec}$, for the tetanic stimulation (arrowheads) $4 \mathrm{mV}$ and $6 \mathrm{sec}$. the processes. Using anti-synapsin antibody, our laboratory established in an earlier study that B2 neurons express synapsin (Malkinson et al. 2006). In addition, Humeau et al. (2001) demonstrated that intracellular injection of anti-synapsin antibody to cholinergic Aplysia neurons led to an increased rate of synaptic depression and the disappearance of PTP. In the sensorymotor synapse formed between cultured Aplysia neurons synapsin overexpression affects the depression rate and 5HT-induced facilitation of depressed synapses (Fioravante et al. 2007). Thus, it was reasonable to assume that, as in other systems (Fdez and Hilfiker 2006), synapsins play a role in regulating the availability of vesicle stores in Aplysia neurons.

Aplysia synapsin (apSyn) has been shown to contain phosphorylation sites for PKA, MAPK, and CaMKI/IV (Angers et al. 2002). Based on the above, we next examined whether inhibition of these kinases affects basal synaptic transmission, frequency facilitation, and PTP of the B2-MCn synapse. To inhibit PKA we used KT5720, which was demonstrated to inhibit 5HT-induced phosphorylation and subcellular redistribution of apSyn (Angers et al. 2002). Ten micromolar of KT5720 had no significant effect on the basal transmission (the first mean control EPSP was $1.35 \pm$ $0.06 \mathrm{mV}, n=16$, and $1.55 \pm 0.47 \mathrm{mV}, n=7$ in KT5720, $P>0.05$ ) and on facilitation (Fig. $7 \mathrm{~B} ; F_{(49,147)}=1, P=0.482$; repeated measures one-way ANOVA, $n=5$ ). However, in the presence of 10 $\mu \mathrm{M}$ KT5 720 the PTP was attenuated by $54.3 \%$ (Fig. 8C; control PTP peak amplitude was $275 \% \pm 47 \%$ of the mean pre-tetanic value, $n=6$, and $180 \% \pm 37 \%$ in KT5720, $n=5, P<0.05$, independent samples $t$-test,). When the PTP was tested in the presence of both calpeptin and KT5720, the PTP was totally abolished (Fig. 8C; $n=$ $6, P<0.01$, independent samples $t$-test). In fact, under these conditions tetanic stimulation led to reduction of the EPSP to $50 \% \pm$ $8 \%$ of the average EPSP amplitude prior to the tetanic stimulus.

Next we tested the effect of U0126, a specific inhibitor of MAP kinase kinase (MEK), which phosphorylates and activates MAPK (Favata et al. 1998; Chin et al. 2002), on basal synaptic transmission. U0126 was shown to block 5HT-induced apSyn phosphorylation and its redistribution in Aplysia sensory neurons (Angers et al. 2002). We found that, in the presence of $20 \mu \mathrm{M}$ U0126, action potentials in B2 neurons were significantly broadened (Fig. 7C; the mean time from the peak of the spike to when membrane potential had declined to $33 \%$ of peak amplitude was $2.62 \pm 0.31 \mathrm{msec}$ in ASW, $n=8$, and $24 \pm 4 \mathrm{msec}$ in U0126, $n=7$, $P<0.01$, independent samples $t$-test). As a result of spike broadening, the EPSP's amplitude increased (first mean control EPSP was $1.88 \pm 0.4 \mathrm{mV}, n=6$, and $5.29 \pm 1.25 \mathrm{mV}, n=6$ in U0126, $P<0.05)$. Stimulation of B2 neuron in the presence of U0126 at $2 \mathrm{~Hz}$ resulted in an increased depression rate relative to control experiments (Fig. 7B; $F_{(49,147)}=1.56, P=0.049$; repeated measures one-way ANOVA, $n=5)$. The increased depression rate by U0126 was reported previously at the sensory-motor synapse (Chin et al. 2002). The mean PTP peak amplitude was attenuated in U0126 by $91 \%$ to $117 \% \pm 15 \%$ (Fig. $8 \mathrm{C} ; n=7, P<0.05$, independent samples $t$-test). Tetanic stimulation of B2-MCn synapses in calpeptin and U0126 together resulted in PTD (first post-tetanic EPSP decreased to $76 \% \pm 13 \%$ of the average pre-tetanic EPSP amplitude, $n=5, P<$ 0.05 , independent samples $t$-test).

Finally, to inhibit the $\mathrm{Ca}^{2+} /$ calmodulin-dependent protein kinases we used KN-93 (Sumi et al. 1991; Corcoran and Means 2001), which impairs PTP in other synapses (Jin and Hawkins 2003; Fiumara et al. 2007). We found that preincubation of the neurons in $10 \mu \mathrm{M} \mathrm{KN}-93$ for $15 \mathrm{~min}$ had no effect on the basal level of transmission (first mean control EPSP was $1.35 \pm 0.06 \mathrm{mV}$, $n=16$, and $1.29 \pm 0.13 \mathrm{mV}, n=5$ in $\mathrm{KN}-93, P>0.05)$. Stimulation of the presynaptic B2 neuron at $2 \mathrm{~Hz}$, in the presence of $\mathrm{KN}-93$, resulted in release kinetics that did not differ from the control (Fig. $7 \mathrm{~B} ; F_{(49,147)}=0.62, P=0.97$; repeated measures one-way ANOVA, 
$n=4)$. However, $10 \mu \mathrm{M}$ KN-93 significantly attenuated the PTP peak amplitude to $198 \% \pm 21 \%, 51.4 \%$ of the peak PTP in control experiments (Fig. 8C; $n=5, P<0.05$, independent samples $t$-test).

In the presence of both $100 \mu \mathrm{M}$ calpeptin and $10 \mu \mathrm{M}$ KN-93 the tetanic stimulus was followed by synaptic depression to $69 \% \pm$ $5 \%$ of the average EPSP amplitude prior to the tetanic stimulus $(n=$ $5, P<0.01$, independent samples $t$-test). These results indicate that inhibition of PKA, MAPK, or CaMKs attenuates PTP in B2-MCn synapse, and combination of each of these inhibitors with calpain inhibitor calpeptin results in PTD.

Theoretically, calpeptin and the kinases inhibitors used could exert their effects on PTP by altering the dynamics of the $\left[\mathrm{Ca}^{2+}\right]_{i}$ transients. To examine this possibility we loaded B2 neurons with the Ca indicator rhod-2 and measured the fluorescent changes in neurites by line scans. Care was taken to carry out the experiments such that saturation of the indicator will not mask the results. The effects of calpeptin $(100 \mu \mathrm{M}), \mathrm{KN}-93(10 \mu \mathrm{M})$, U0126 $(20 \mu \mathrm{M})$, and KT5720 $(10 \mu \mathrm{M})$ were tested. We found that the rise and decay time of the $\left[\mathrm{Ca}^{2+}\right]_{\mathrm{i}}$ transient generated by identical tetanic stimuli or the peak fluorescent amplitudes were not altered by the drugs, except for calpeptin, which facilitated the rise time (Table 1).

\section{Discussion}

The present study demonstrates that the Ca-activated proteasecalpain serves as a critical component in the cascade that links activity-dependent elevation of $\left[\mathrm{Ca}^{2+}\right]_{i}$ in the presynaptic terminal, facilitation, and PTP. Our study revealed that the calpain inhibitor, calpeptin, transforms a facilitating synapse to a depressing one and reduces PTP by $80.6 \%$. Inhibition of PKA, MAPK, and CaMKs, which presumably inhibits phosphorylation of apSyn, reduces the PTP value by $54.3 \%, 91 \%$, and $44 \%$, respectively. When each of these inhibitors is applied together with calpeptin, tetanic train of stimuli leads to depression of the EPSP amplitude in comparison to the pre-tetanic value. We therefore suggest that, at the B2-MCn synapse, tetanic stimulation activates in parallel two pathways that underlay PTP: a calpain-dependent path and a PKA/CaMKs/MAPK/synapsin path.

PTP is a form of short-term synaptic enhancement. Although postsynaptic mechanisms have been shown to play some role in PTP of Aplysia sensory-motor synapse (Bao et al. 1997; Schaffhausen et al. 2001), it mostly relies on Ca accumulation during tetanic stimulation at the presynaptic terminals (for review, see Zucker and Regehr 2002). It was proposed that Ca triggers vesicles mobilization from the reserve pool to the ready-releasable pool and that this process is controlled by synapsins (Humeau et al. 2001; Zucker and Regehr 2002; Fioravante et al. 2007). Other mechanisms such as modulation of the efficacy of release mechanisms and switching on individual synaptic release sites were also proposed to contribute to PTP (Royer et al. 2000; Zhao and Klein 2004; Humeau et al. 2007; Korogod et al. 2007).
Synapsins are major but not exclusive synaptic substrates of PKA, MAPK, and CaMKs I, II, and IV (Llinas et al. 1991; Jovanovic et al. 1996; Chi et al. 2001, 2003; Fiumara et al. 2004; Menegon et al. 2006). Indeed, inhibition of PKA (Fiumara et al. 2007), MAPK (Jin and Hawkins 2003), or CaMKs (Jin and Hawkins 2003; Fiumara et al. 2007) attenuates PTP. Moreover, synapsin neutralization by anti-synapsin antibodies reduced PTP at Aplysia cholinergic synapses (Humeau et al. 2001).

Aplysia synapsin contains consensus sequences for PKA and CaMK I/IV (domain A) and for MAPK (Angers et al. 2002) and is phosphorylated in vitro by these kinases (Fiumara et al. 2004). Although we have not demonstrated directly the involvement of synapsins in PTP at the B2-MCn synapse, the fact that the inhibitors of all three kinases (PKA, MAPK, and CaMKs) affected the PTP suggests that synapsin phosphorylation is involved in PTP. The occurrence of PTD, which was measured when both calpain activity and synapsin phosphorylation were blocked, is consistent with the hypothesis that tetanic stimulation triggers vesicles mobilization by both calpain activation and synapsin phosphorylation. When both pathways are blocked, the PTP is transformed to PTD, suggesting that under these conditions following tetanic stimulation either the store of synaptic vesicles is exhausted to below control levels or part of the release machinery that operates under control conditions ceases to operate.

PTD was demonstrated in Aplysia cholinergic synapse injected with anti-synapsin antibodies (Humeau et al. 2001). The authors suggested that the reserve pool of presynaptic vesicles is depleted under conditions of synapsin neutralization and as a result the readyreleasable pool cannot be replenished at a sufficient rate during tetanic stimulus, leading to its exhaustion and transient PTD.

\section{Possible calpain substrates that regulate facilitation and PTP}

Whereas the mechanisms by which synapsin phosphorylation and dephosphorylation regulate synaptic release have been studied extensively (Llinas et al. 1985; Lin et al. 1990; Hosaka et al. 1999; Chi et al. 2001, 2003; Fdez and Hilfiker 2006; Evergren et al. 2007), the mechanisms by which calpain activation regulates release processes are just beginning to emerge.

The calpain super family of Ca-dependent papain-like cysteine proteases (Guroff 1964) functions to post-translationally modify over 100 substrates by partial proteolysis rather than by complete proteolytic digestion (Carragher and Frame 2002; Evans and Turner 2007). Consequently, the calpain super family has been implicated in playing central roles in a large number of physiological and pathological processes (Goll et al. 2003; Wu and Lynch 2006; Evans and Turner 2007; Bevers and Neumar 2008). Regarding neurotransmitter and neurohormone release, calpains were suggested to regulate (1) mobilization of vesicles to release sites, (2) membrane cycling, and (3) directly the molecular apparatus of vesicle release.

Table 1. Effects of calpain and kinases inhibitors on Ca transients generated by tetanic stimuli

\begin{tabular}{|c|c|c|c|c|c|c|}
\hline & \multicolumn{2}{|c|}{$\Delta \mathrm{F} / \mathrm{F} 0$} & \multicolumn{2}{|c|}{ Rise time (sec) } & \multicolumn{2}{|c|}{ Decay time (sec) } \\
\hline & Drug & Control & Drug & Control & Drug & Control \\
\hline Calpeptin $n=5$ & 2.5 & 2.7 & $0.16^{\mathrm{a}}$ & 0.26 & 2.67 & 2.44 \\
\hline KT5720 $n=3$ & 3.42 & 3.82 & 0.28 & 0.27 & 2.61 & 2.54 \\
\hline $\mathrm{KN}-93 n=4$ & 3.59 & 3.55 & 0.22 & 0.21 & 1.47 & 1.41 \\
\hline$\cup 0126 n=4$ & 2.91 & 3.79 & 0.21 & 0.26 & 2.27 & 2.24 \\
\hline
\end{tabular}

Cultured B2 neurons were microinjected with rhod-2 pentapotassium and the fluorescent intensity within the neurites was monitored by the line scan mode of the confocal system while the neuron was intracellularly stimulated at $20 \mathrm{~Hz}$ for $2 \mathrm{sec}$. First, the neuron was stimulated in ASW and after a rest period of $5 \mathrm{~min}$ in the presence of the inhibitor.

${ }^{\mathrm{a}} P<0.05$; paired samples $t$-test. 
It was suggested that, in neuroendocrine cells (INS-1), calpain 1 frees tethered granules from the actin microfilaments, thus allowing them to move to the plasma membrane and then fuse (Ort et al. 2001). This conclusion was recently supported by demonstrating that calpain cleaves synapsin (Murrey et al. 2006). In addition, the calpain substrate, spectrin, which serves as a submembrane skeletal component (Siman et al. 1984; Seubert et al. 1987), is often preferentially localized to synapses, and was shown to bind small synaptic vesicles via synapsin I (Sikorski and Goodman 1991; Sikorski et al. 1991). Antibodies directed against 15 amino acids of the synapsin I binding domain of $\beta$-spectrin blocked synaptic transmission in culture hippocampal neurons (Sikorski et al. 2000; Zimmer et al. 2000). Elevation of the free intracellular Ca concentration in cultured Aplysia neurons leads to submembrane spectrin cleavage, followed by fusion of vesicles with the plasma membrane at this location (Erez et al. 2007; Erez and Spira 2008). These processes are inhibited by the calpain inhibitor, calpeptin (Gitler and Spira 1998). Furthermore, in our previous paper (Khoutorsky and Spira 2005), we presented evidence suggesting that, in the sensory-motor synapse of Aplysia, vesicle mobilization is a calpain-dependent process.

Calpain 1 and 2 were found to be linked to coated vesicles in K562 cells and pituitary cells (Sato et al. 1995; Ohkawa et al. 2000). It was suggested that in mucosa cells calpain dissociates $\beta$-coat protein and triggers vesicular trafficking (Hata et al. 2006). Thus, it is conceivable that calpain participates in the process of membrane cycling and thereby may affect the rates of vesicle refilling.

A number of the SNARE complex proteins including syntaxin1, VAMP-2, and SNAP-25 are cleaved by calpain 10 (Zimmerman et al. 1999; Rutledge and Whiteheart 2002; Marshall et al. 2005), a process that was hypothesized to directly induce exocytosis (Evans and Turner 2007). In contrast, calpain activation was suggested to suppress $\mathrm{Ca}^{2+}$-dependent glutamate release from cerebellar granule cells (Ando et al. 2005).

The experiments presented here are consistent with the hypothesis that inhibition of calpain inhibits activity-dependent mobilization of a fraction of vesicles from the reserve pool to the ready-releasable vesicle store. However, the experiments do not allow us to unequivocally differentiate among the various mechanisms discussed above.

\section{Possible mechanisms by which calpain and synapsin regulate facilitation and PTP}

Since confocal microscope imaging of membrane cycling using conventional procedures of loading and unloading of FM dyes (Betz and Bewick 1992) did not reveal any sign of localized membrane retrieval in our preparation (data not shown), we assume that in the synapse formed by cultured B2 and MCn the refilling processes are mainly dependent on the supply of anterogradely transported vesicles from the Golgi apparatus. Based on this assumption, and in line with the literature, we provide below some working models to account for a possible role of calpain in regulating the refilling of the ready-releasable pool of neurotransmitter-containing vesicles.

(1) Action potentials delivered to the presynaptic B2 neurons at low frequencies evoke low quantal content EPSPs. At slow stimulation rates, constitutive refilling is sufficient to maintain the readyreleasable pool at a steady state. (2) Repetitive stimulation at higher frequencies elevates $\left[\mathrm{Ca}^{2}\right]_{\mathrm{i}}$ to higher levels that are sufficient to activate calpain but are not high enough to activate CaMKs/PKA/ MAPK/synapsin pathway. (3) Active calpain cleaves substrates that impede the mobilization of vesicles from nonreleasable store into a ready-releasable store. (4) Following tetanic stimulation, both calpain and CaMKs/PKA/MAPK are activated. The elevation of the $\left[\mathrm{Ca}^{2+}\right]_{\mathrm{i}}$ during tetanic stimulation may be sufficient to induce $\mathrm{Ca}^{2+} /$ calmodulin-mediated activation of adenylyl cyclase which in turn activates several signal transduction pathways, including the MAPK and PKA pathways (Ferguson and Storm 2004; Menegon et al. 2006). CaMKs/PKA/MAPK phosphorylate synapsins and thereby release tethered vesicles so that they are free to move and replenish the ready-releasable pool. Since the inhibition of both calpain and each of apSyn phosphorylating kinases (PKA/MAPK/CaMKs) leads to PTD, it is reasonable to assume that there are no major additional mechanisms that restrict vesicle mobilization to the ready-releasable pool. Interestingly, it was previously reported that CaM kinase IIa is proteolysed by calpain to produce an active catalytic fragment lacking its calmodulin-binding auto-inhibitory domains (Yoshimura et al. 1996; Hajimohammadreza et al. 1997). Thus, CaM kinases activation by calpain may amplify the contribution of calpain to vesicle mobilization.

Note that the proposed model assumes that both calpain activation and synapsin phosphorylation conceptually operate to remove molecular barriers that impede vesicle mobilization rather than in regulating vesicle motility. The speed and directionality of vesicle translocation is probably controlled by other factors including protein kinase C. In cultured Aplysia sensory-motoneuron synapse, experimental manipulations, that activate a Ca-independent PKC, lead to vesicle mobilization following the depletion of the ready-releasable vesicle pool by trains of action potentials (Braha et al. 1990; Ghirardi et al. 1992; Sossin et al. 1993; Byrne and Kandel 1996; Manseau et al. 2001). This replenishment is inhibited by calpain inhibitors, indicating that calpain activation precedes to allow the action of PKC (Khoutorsky and Spira 2005). Finally, it should be noted that, in contrast to reports implying that calpain 10 directly regulates the SNARE complex by proteolysis of SNAP-25 in pancreatic cells (Marshall et al. 2005; Evans and Turner 2007), in the synaptic junction studied here calpain inhibitors interfere with facilitation and PTP but do not play a role in events that underlie the fusion of docked vesicles with the plasma membrane, as evidenced by the observation that calpain inhibitors do not block the release at low stimulation frequencies.

\section{Materials and Methods}

\section{Cell cultures}

Buccal neuron 2 (B2) from the buccal ganglia and metacerebral neuron (MCn) from the cerebral ganglia of juvenile Aplysia californica (supplied by the University of Miami) were cocultured, as described by Schacher and Proshansky (1983). Briefly, animals were anesthetized by injection of isotonic $\mathrm{MgCl} 2$ solution. The ganglia were isolated and incubated for $1.5-3 \mathrm{~h}$ in $1 \%$ protease (type IX, Sigma) at $34^{\circ} \mathrm{C}$. Next, the ganglia were desheathed, and the cell bodies of their neurons with their axons were pulled out with sharp micropipettes and placed on poly-L-lysine-coated (Sigma) glass-bottomed culture dishes. The culture medium consisted of 10\% filtered hemolymph from Aplysia faciata collected along the Mediterranean coast, and L-15 (Gibco-BRL) supplemented for marine species. Twenty-four hours after plating, the dishes were transferred to an $18^{\circ} \mathrm{C}$ incubator. The experiments were performed 2-4 d after plating, at room temperature (between $20^{\circ} \mathrm{C}$ and $24^{\circ} \mathrm{C}$ ) after replacing the culture medium with artificial sea water (ASW) composed of $\mathrm{NaCl} 460 \mathrm{mM}, \mathrm{KCL} 10 \mathrm{mM}, \mathrm{CaCl}_{2}$ $11 \mathrm{mM}, \mathrm{MgCl}_{2} 55 \mathrm{mM}$, and HEPES (N-[2-hydroxyethyl]piperazine$\mathrm{N}^{\prime}$-2ethanesulfonic acid, Sigma) $10 \mathrm{mM}$, adjusted to $\mathrm{pH}$ 7.6.

\section{Electrophysiology}

B2 neurons were stimulated intracellularly with a 10-20-mseclong depolarizing current pulse (Axoclamp-2A; Axon Instruments) using a sharp 5-10 $\mathrm{M} \Omega$ glass microelectrode filled with $2 \mathrm{M} \mathrm{KCl}$. Postsynaptic potentials (PSPs) were recorded intracellularly from the cell body while holding the postsynaptic transmembrane potential at approximately $-60 \mathrm{mV}$. Signals were digitally recorded by means of a Digidata 1322A interface (Axon Instruments) and analyzed with Clampfit software (Axon Instruments). 


\section{Drugs}

Calpeptin (Z-Leu-Nle-CHO) and MG132 (Z-Leu-Leu-Leu-CHO, Calbiochem) were prepared as $50 \mathrm{mM}$ stock solutions in DMSO (Sigma) and diluted to the final concentration just before the experiments. KN-93 (2-[N-(2-hydroxyethyl)-N-(4-methoxybenzenesulfonyl)] amino-N-(4-chlorocinnamyl)-N-ethylbenzylamine) and clasto-lactacystin $\beta$-lactone (Calbiochem) were prepared as $10 \mathrm{mM}$ stock solution in DMSO. U0126 (1,4-diamino-2,3-dicyano-1,4bis[2-aminophenylthio] butadiene) and KT5720 ([9S,10S,12R] -2,3,9,10,11,12-Hexahydro-10-hydroxy-9-methyl-1-oxo-9,12-epoxy1H-diindolo[1,2,3-fg: $\left.3^{\prime}, 2^{\prime}, 1-k 1\right]$ pyrrolo[3,4-i] [1,6]benzodiazocine10 -carboxylic acid hexyl ester, Sigma) were prepared as $10 \mathrm{mM}$ and $5 \mathrm{mM}$ stock solutions, respectively, in DMSO. In control experiments, bath application of the vehicle solution composed of $0.2 \%$ DMSO in ASW had no effect.

\section{Ca imaging}

The system used for confocal imaging consisted of an Olympus microscope IX70 and a Bio-Rad Radiance 2000/AGR-3 confocal imaging system. The objective used was an Olympus planApo $60 \times$ $1.4 \mathrm{NA}$ oil objective. The images were collected and processed using LaserSharp and LaserPix BioRad software, respectively. For the experiments, fluo- $410 \mathrm{mM}$ or rhod- $210 \mathrm{mM}$ (pentapotassium salt, Invitrogen) in $0.5 \mathrm{M} \mathrm{KCl}$ was microinjected into the neurons by pressure. Imaging was performed after the dye had equilibrated throughout the main axon and the small neurites $(\sim 30 \mathrm{~min}$ after the injection). Fluo-4 was excited with $488 \mathrm{~nm}$ (Argon laser), the emission collected at 500-560 nm (Malkinson and Spira 2006). Rhod-2 was excited with $543 \mathrm{~nm}$ (Green HeNe laser) and the emission collected at $605 \pm 37.5 \mathrm{nM}$.

\section{ACh application}

ACh was pressure-ejected through a $2-\mu \mathrm{m}$ tip diameter pipette. The pressure pulse duration varied from 10 to $40 \mathrm{msec}$ and from 1 to 2 p.s.i. $(7-14 \mathrm{kPa})$.

\section{Neuron staining}

To image the morphological relations between the neurons, B2 and MCn were pressure-injected with carboxifluorescein $(0.44 \mathrm{M}$ in $\mathrm{KOH}$ stock solution diluted $1 / 100$ in $0.5 \mathrm{M} \mathrm{KCl}$ ) and Alexa Fluor 555 hydrazide (10 $\mathrm{mM}$ in $0.5 \mathrm{M} \mathrm{KCl}$, Invitrogen), respectively, on the third day after plating. Then, the neurons were imaged using 488- and 543-nm laser lines for excitation and the emitted lights were collected using the standard sets of fluorescein and rhodamine filters. The varicosities of $\mathrm{B} 2$ neuron on the main axon of the MCn were visually identified and counted.

\section{Statistical analysis}

The effects of various treatments are presented as the percentage change of EPSP amplitude after treatment with respect to the initial EPSP amplitude pre-treatment. All the data are presented as mean \pm SEM. Statistical analysis was performed with the software package SPSS 14.0 using repeated measures one-way ANOVA, independent samples $t$-test, and the paired samples $t$-test. In all instances, a value of $P<0.05$ was considered as statistically significant.

To analyze the quantal content, we used the coefficient of variance method in which $m=\operatorname{EPSP}^{2} / \sigma^{2}$, where $m$ is the quantal content, $\mathrm{EPSP}^{2}$ is the squared mean EPSP amplitude, and $\sigma$ is the variance of the EPSP's amplitude. To calculate the mean EPSP amplitude and the variance, at least 500 consecutive EPSPs per experiment were evoked at a rate of $1 \mathrm{~Hz}$ and analyzed using Clampfit (Axon Instruments) and Origin 7 (OriginLab Corporation) software.

\section{Acknowledgments}

This study was supported by the US-Israel Binational Science Research Foundation, grant number 2003152. Part of the work was done at the Charles E. Smith Family and Professor Joel Elkes Laboratory for Psychobiology. M.E.S. is the Levi DeViali Professor in Neurobiology.

\section{References}

Ando, K., Kudo, Y., and Takahashi, M. 2005. Negative regulation of neurotransmitter release by calpain: A possible involvement of specific SNAP-25 cleavage. J. Neurochem. 94: 651-658.

Angers, A., Fioravante, D., Chin, J., Cleary, L.J., Bean, A.J., and Byrne, J.H. 2002. Serotonin stimulates phosphorylation of Aplysia synapsin and alters its subcellular distribution in sensory neurons. J. Neurosci. 22: 5412-5422.

Bailey, C.H. and Kandel, E.R. 2008. Synaptic remodeling, synaptic growth and the storage of long-term memory in Aplysia. Prog. Brain Res. 169: 179-198.

Bao, J.X., Kandel, E.R., and Hawkins, R.D. 1997. Involvement of pre- and postsynaptic mechanisms in posttetanic potentiation at Aplysia synapses. Science 275: 969-973.

Betz, W.J. and Bewick, G.S. 1992. Optical analysis of synaptic vesicle recycling at the frog neuromuscular junction. Science 255: 200-203.

Bevers, M.B. and Neumar, R.W. 2008. Mechanistic role of calpains in postischemic neurodegeneration. J. Cereb. Blood Flow Metab. 28: 655673.

Blatow, M., Caputi, A., Burnashev, N., Monyer, H., and Rozov, A. 2003. Ca ${ }^{2+}$ buffer saturation underlies paired pulse facilitation in calbindin-D28kcontaining terminals. Neuron 38: 79-88.

Braha, O., Dale, N., Hochner, B., Klein, M., Abrams, T.W., and Kandel, E.R. 1990. Second messengers involved in the two processes of presynaptic facilitation that contribute to sensitization and dishabituation in Aplysia sensory neurons. Proc. Natl. Acad. Sci. 87: 2040-2044.

Brodin, L., Low, P., Gad, H., Gustafsson, J., Pieribone, V.A., and Shupliakov, O. 1997. Sustained neurotransmitter release: New molecular clues. Eur. J. Neurosci. 9: 2503-2511.

Byrne, J.H. and Kandel, E.R. 1996. Presynaptic facilitation revisited: State and time dependence. J. Neurosci. 16: 425-435.

Carragher, N.O. and Frame, M.C. 2002. Calpain: A role in cell transformation and migration. Int. J. Biochem. Cell Biol. 34: 1539-1543.

Castellucci, V.F. and Kandel, E.R. 1974. A quantal analysis of the synaptic depression underlying habituation of the gill-withdrawal reflex in Aplysia. Proc. Natl. Acad. Sci. 71: 5004-5008.

Ceccarelli, B., Hurlbut, W.P., and Mauro, A. 1973. Turnover of transmitter and synaptic vesicles at the frog neuromuscular junction. J. Cell Biol. 57: 499-524.

Chi, P., Greengard, P., and Ryan, T.A. 2001. Synapsin dispersion and reclustering during synaptic activity. Nat. Neurosci. 4: 1187-1193.

Chi, P., Greengard, P., and Ryan, T.A. 2003. Synaptic vesicle mobilization is regulated by distinct synapsin I phosphorylation pathways at different frequencies. Neuron 38: 69-78.

Chin, J., Angers, A., Cleary, L.J., Eskin, A., and Byrne, J.H. 2002. Transforming growth factor $\beta 1$ alters synapsin distribution and modulates synaptic depression in Aplysia. J. Neurosci. 22: 1-6.

Corcoran, E.E. and Means, A.R. 2001. Defining Ca ${ }^{2+} /$ calmodulin-dependent protein kinase cascades in transcriptional regulation. J. Biol. Chem. 276: 2975-2978.

Del Castillo, J. and Katz, B. 1954. Quantal components of the end-plate potential. J. Physiol. 124: 560-573.

Dick, L.R., Cruikshank, A.A., Grenier, L., Melandri, F.D., Nunes, S.L., and Stein, R.L. 1996. Mechanistic studies on the inactivation of the proteasome by lactacystin: A central role for clasto-lactacystin $\beta$-lactone. J. Biol. Chem. 271: 7273-7276.

Dittman, J.S. and Regehr, W.G. 1998. Calcium dependence and recovery kinetics of presynaptic depression at the climbing fiber to Purkinje cell synapse. J. Neurosci. 18: 6147-6162.

Erez, H. and Spira, M.E. 2008. Local self-assembly mechanisms underlie the differential transformation of the proximal and distal cut axonal ends into functional and aberrant growth cones. J. Comp. Neurol. 507: 10191030.

Erez, H., Malkinson, G., Prager-Khoutorsky, M., De Zeeuw, C.I., Hoogenraad, C.C., and Spira, M.E. 2007. Formation of microtubulebased traps controls the sorting and concentration of vesicles to restricted sites of regenerating neurons after axotomy. J. Cell Biol. 176: 497-507.

Evans, J.S. and Turner, M.D. 2007. Emerging functions of the calpain superfamily of cysteine proteases in neuroendocrine secretory pathways. J. Neurochem. 103: 849-859.

Evergren, E., Benfenati, F., and Shupliakov, O. 2007. The synapsin cycle: A view from the synaptic endocytic zone. J. Neurosci. Res. 85: 26482656.

Favata, M.F., Horiuchi, K.Y., Manos, E.J., Daulerio, A.J., Stradley, D.A., Feeser, W.S., Van Dyk, D.E., Pitts, W.J., Earl, R.A., Hobbs, F. , et al.1998. Identification of a novel inhibitor of mitogen-activated protein kinase kinase. J. Biol. Chem. 273: 18623-18632.

Fdez, E. and Hilfiker, S. 2006. Vesicle pools and synapsins: New insights into old enigmas. Brain Cell Biol. 35: 107-115. 
Felmy, F., Neher, E., and Schneggenburger, R. 2003. Probing the intracellular calcium sensitivity of transmitter release during synaptic facilitation. Neuron 37: 801-811.

Fenteany, G., Standaert, R.F., Lane, W.S., Choi, S., Corey, E.J., and Schreiber, S.L. 1995. Inhibition of proteasome activities and subunit-specific amino-terminal threonine modification by lactacystin. Science 268: 726-731.

Ferguson, G.D. and Storm, D.R. 2004. Why calcium-stimulated adenylyl cyclases? Physiology (Bethesda) 19: 271-276.

Figueiredo-Pereira, M.E., Banik, N., and Wilk, S. 1994. Comparison of the effect of calpain inhibitors on two extralysosomal proteinases: The multicatalytic proteinase complex and m-calpain. J. Neurochem. 62: 1989-1994.

Fioravante, D., Liu, R.Y., Netek, A.K., Cleary, L.J., and Byrne, J.H. 2007. Synapsin regulates basal synaptic strength, synaptic depression, and serotonin-induced facilitation of sensorimotor synapses in Aplysia. J. Neurophysiol. 98: 3568-3580.

Fiumara, F., Giovedi, S., Menegon, A., Milanese, C., Merlo, D., Montarolo, P.G., Valtorta, F., Benfenati, F., and Ghirardi, M. 2004. Phosphorylation by cAMP-dependent protein kinase is essential for synapsin-induced enhancement of neurotransmitter release in invertebrate neurons. J. Cell Sci. 117: 5145-5154.

Fiumara, F., Milanese, C., Corradi, A., Giovedi, S., Leitinger, G., Menegon, A., Montarolo, P.G., Benfenati, F., and Ghirardi, M. 2007. Phosphorylation of synapsin domain A is required for post-tetanic potentiation. J. Cell Sci. 120: 3228-3237.

Gaffield, M.A. and Betz, W.J. 2007. Synaptic vesicle mobility in mouse motor nerve terminals with and without synapsin. J. Neurosci. 27: 13691-13700.

Geiger, J.R. and Jonas, P. 2000. Dynamic control of presynaptic $\mathrm{Ca}^{2+}$ inflow by fast-inactivating $\mathrm{K}^{+}$channels in hippocampal mossy fiber boutons. Neuron 28: 927-939.

Ghirardi, M., Braha, O., Hochner, B., Montarolo, P.G., Kandel, E.R., and Dale, N. 1992. Roles of PKA and PKC in facilitation of evoked and spontaneous transmitter release at depressed and nondepressed synapses in Aplysia sensory neurons. Neuron 9: 479-489.

Gingrich, K.J. and Byrne, J.H. 1985. Simulation of synaptic depression, posttetanic potentiation, and presynaptic facilitation of synaptic potentials from sensory neurons mediating gill-withdrawal reflex in Aplysia. J. Neurophysiol. 53: 652-669.

Gitler, D. and Spira, M.E. 1998. Real time imaging of calcium-induced localized proteolytic activity after axotomy and its relation to growth cone formation. Neuron 20: 1123-1135.

Gitler, D. and Spira, M.E. 2002. Short window of opportunity for calpain induced growth cone formation after axotomy of Aplysia neurons. J. Neurobiol. 52: 267-279.

Goll, D.E., Thompson, V.F., Li, H., Wei, W., and Cong, J. 2003. The calpain system. Physiol. Rev. 83: 731-801.

Gover, T.D., Jiang, X.Y., and Abrams, T.W. 2002. Persistent, exocytosisindependent silencing of release sites underlies homosynaptic depression at sensory synapses in Aplysia. J. Neurosci. 22: 19421955.

Greengard, P., Valtorta, F., Czernik, A.J., and Benfenati, F. 1993. Synaptic vesicle phosphoproteins and regulation of synaptic function. Science 259: 780-785.

Guroff, G. 1964. A neutral, calcium-activated proteinase from the soluble fraction of rat brain. J. Biol. Chem. 239: 149-155.

Habets, R.L. and Borst, J.G. 2005. Post-tetanic potentiation in the rat calyx of Held synapse. J. Physiol. 564: 173-187.

Habets, R.L. and Borst, J.G. 2007. Dynamics of the readily releasable pool during post-tetanic potentiation in the rat calyx of Held synapse. $J$. Physiol. 581: 467-478.

Hajimohammadreza, I., Raser, K.J., Nath, R., Nadimpalli, R., Scott, M., and Wang, K.K. 1997. Neuronal nitric oxide synthase and calmodulindependent protein kinase II $\alpha$ undergo neurotoxin-induced proteolysis. J. Neurochem. 69: 1006-1013.

Hata, S., Koyama, S., Kawahara, H., Doi, N., Maeda, T., Toyama-Sorimachi, N., Abe, K., Suzuki, K., and Sorimachi, H. 2006. Stomach-specific calpain, nCL-2, localizes in mucus cells and proteolyzes the $\beta$-subunit of coatomer complex, $\beta$-COP. J. Biol. Chem. 281: 11214-11224.

Heuser, J.E. and Reese, T.S. 1973. Evidence for recycling of synaptic vesicle membrane during transmitter release at the frog neuromuscular junction. J. Cell Biol. 57: 315-344.

Hilfiker, S., Schweizer, F.E., Kao, H.T., Czernik, A.J., Greengard, P., and Augustine, G.J. 1998. Two sites of action for synapsin domain E in regulating neurotransmitter release. Nat. Neurosci. 1: 29-35.

Hilfiker, S., Pieribone, V.A., Czernik, A.J., Kao, H.T., Augustine, G.J., and Greengard, P. 1999. Synapsins as regulators of neurotransmitter release. Philos. Trans. R. Soc. Lond. B Biol. Sci. 354: 269-279.

Hosaka, M., Hammer, R.E., and Sudhof, T.C. 1999. A phospho-switch controls the dynamic association of synapsins with synaptic vesicles. Neuron 24: $377-387$.
Hosoi, N., Sakaba, T., and Neher, E. 2007. Quantitative analysis of calciumdependent vesicle recruitment and its functional role at the calyx of Held synapse. J. Neurosci. 27: 14286-14298.

Humeau, Y., Doussau, F., Vitiello, F., Greengard, P., Benfenati, F., and Poulain, B. 2001. Synapsin controls both reserve and releasable synaptic vesicle pools during neuronal activity and short-term plasticity in Aplysia. J. Neurosci. 21: 4195-4206.

Humeau, Y., Doussau, F., Popoff, M.R., Benfenati, F., and Poulain, B. 2007 Fast changes in the functional status of release sites during short-term plasticity: Involvement of a frequency-dependent bypass of Rac at Aplysia synapses. J. Physiol. 583: 983-1004.

Jackson, M.B., Konnerth, A., and Augustine, G.J. 1991. Action potential broadening and frequency-dependent facilitation of calcium signals in pituitary nerve terminals. Proc. Natl. Acad. Sci. 88: 380-384.

Jin, I. and Hawkins, R.D. 2003. Presynaptic and postsynaptic mechanisms of a novel form of homosynaptic potentiation at Aplysia sensory-motor neuron synapses. J. Neurosci. 23: 7288-7297.

Jovanovic, J.N., Benfenati, F., Siow, Y.L., Sihra, T.S., Sanghera, J.S., Pelech, S.L., Greengard, P., and Czernik, A.J. 1996. Neurotrophins stimulate phosphorylation of synapsin I by MAP kinase and regulate synapsin I-actin interactions. Proc. Natl. Acad. Sci. 93: 3679-3683.

Jovanovic, J.N., Czernik, A.J., Fienberg, A.A., Greengard, P., and Sihra, T.S. 2000. Synapsins as mediators of BDNF-enhanced neurotransmitter release. Nat. Neurosci. 3: 323-329.

Kehoe, J. 1972. Three acetylcholine receptors in Aplysia neurons. J. Physiol. 225: $115-146$.

Kehoe, J. and McIntosh, J.M. 1998. Two distinct nicotinic receptors, one pharmacologically similar to the vertebrate $\alpha 7$-containing receptor, mediate Cl currents in Aplysia neurons. J. Neurosci. 18: 8198-8213.

Khoutorsky, A. and Spira, M.E. 2005. Calcium-activated proteases are critical for refilling depleted vesicle stores in cultured sensory-motor synapses of Aplysia. Learn. Mem. 12: 414-422.

Khoutorsky, A. and Spira, M.E. 2008. Calpain inhibitors alter the excitable membrane properties of cultured Aplysia neurons. J. Neurophysiol. 100: 2784-2793.

Korogod, N., Lou, X., and Schneggenburger, R. 2007. Post-tetanic potentiation critically depends on an enhanced $\mathrm{Ca}^{2+}$ sensitivity of vesicle fusion mediated by presynaptic PKC. Proc. Natl. Acad. Sci. 104: $15923-15928$

Lee, D.H. and Goldberg, A.L. 1998. Proteasome inhibitors: Valuable new tools for cell biologists. Trends Cell Biol. 8: 397-403.

Lin, J.W., Sugimori, M., Llinas, R.R., McGuinness, T.L., and Greengard, P. 1990. Effects of synapsin I and calcium/calmodulin-dependent protein kinase II on spontaneous neurotransmitter release in the squid giant synapse. Proc. Natl. Acad. Sci. 87: 8257-8261.

Llinas, R., McGuinness, T.L., Leonard, C.S., Sugimori, M., and Greengard, P. 1985. Intraterminal injection of synapsin I or calcium/calmodulindependent protein kinase II alters neurotransmitter release at the squid giant synapse. Proc. Natl. Acad. Sci. 82: 3035-3039.

Llinas, R., Gruner, J.A., Sugimori, M., McGuinness, T.L., and Greengard, P. 1991. Regulation by synapsin I and $\mathrm{Ca}^{2+}$-calmodulin-dependent protein kinase II of the transmitter release in squid giant synapse. $J$. Physiol. 436: 257-282.

Malkinson, G. and Spira, M.E. 2006. Calcium concentration threshold and translocation kinetics of EGFP-DOC2B expressed in cultured Aplysia neurons. Cell Calcium 39: 85-93.

Malkinson, G., Fridman, Z.M., Kamber, D., Dormann, A., Shapira, E., and Spira, M.E. 2006. Calcium-induced exocytosis from actomyosin-driven, motile varicosities formed by dynamic clusters of organelles. Brain Cell Biol. 35: 57-73.

Manseau, F., Fan, X., Hueftlein, T., Sossin, W., and Castellucci, V.F. 2001. $\mathrm{Ca}^{2+}$-independent protein kinase C Apl II mediates the serotonininduced facilitation at depressed Aplysia sensorimotor synapses. $J$. Neurosci. 21: 1247-1256.

Marshall, C., Hitman, G.A., Partridge, C.J., Clark, A., Ma, H., Shearer, T.R., and Turner, M.D. 2005. Evidence that an isoform of calpain-10 is a regulator of exocytosis in pancreatic $\beta$-cells. Mol. Endocrinol. 19: 213-224.

Menegon, A., Bonanomi, D., Albertinazzi, C., Lotti, F., Ferrari, G., Kao, H.T., Benfenati, F., Baldelli, P., and Valtorta, F. 2006. Protein kinase Amediated synapsin I phosphorylation is a central modulator of $\mathrm{Ca}^{2+}$ dependent synaptic activity. J. Neurosci. 26: 11670-11681.

Model, P.G., Highstein, S.M., and Bennett, M.V. 1975. Depletion of vesicles and fatigue of transmission at a vertebrate central synapse. Brain Res. 98: $209-228$

Murrey, H.E., Gama, C.I., Kalovidouris, S.A., Luo, W.I., Driggers, E.M., Porton, B., and Hsieh-Wilson, L.C. 2006. Protein fucosylation regulates synapsin $\mathrm{Ia} / \mathrm{Ib}$ expression and neuronal morphology in primary hippocampal neurons. Proc. Natl. Acad. Sci. 103: 21-26.

Nakanishi, K., Zhang, F., Baxter, D.A., Eskin, A., and Byrne, J.H. 1997. Role of calcium-calmodulin-dependent protein kinase II in modulation of sensorimotor synapses in Aplysia. J. Neurophysiol. 78: 409-416. 
Neher, E. and Sakaba, T. 2008. Multiple roles of calcium ions in the regulation of neurotransmitter release. Neuron 59: 861-872.

Ohkawa, K., Takada, K., Asakura, T., Hashizume, Y., Okawa, Y., Tashiro, K., Ueda, J., Itoh, Y., and Hibi, N. 2000. Calpain inhibitor inhibits secretory granule maturation and secretion of GH. Neuroreport 11: 4007-4011.

Ort, T., Voronov, S., Guo, J., Zawalich, K., Froehner, S.C., Zawalich, W., and Solimena, M. 2001. Dephosphorylation of $\beta 2$-syntrophin and $\mathrm{Ca}^{2+} / \mu$ calpain-mediated cleavage of ICA512 upon stimulation of insulin secretion. EMBO J. 20: 4013-4023.

Patil, P.G., Brody, D.L., and Yue, D.T. 1998. Preferential closed-state inactivation of neuronal calcium channels. Neuron 20: 1027-1038.

Pieribone, V.A., Shupliakov, O., Brodin, L., Hilfiker-Rothenfluh, S., Czernik, A.J., and Greengard, P. 1995. Distinct pools of synaptic vesicles in neurotransmitter release. Nature 375: 493-497.

Rosahl, T.W., Geppert, M., Spillane, D., Herz, J., Hammer, R.E., Malenka, R.C., and Sudhof, T.C. 1993. Short-term synaptic plasticity is altered in mice lacking synapsin I. Cell 75: 661-670.

Rosenmund, C. and Stevens, C.F. 1996. Definition of the readily releasable pool of vesicles at hippocampal synapses. Neuron 16: 1197-1207.

Royer, S., Coulson, R.L., and Klein, M. 2000. Switching off and on of synaptic sites at Aplysia sensorimotor synapses. J. Neurosci. 20: 626-638.

Rutledge, T.W. and Whiteheart, S.W. 2002. SNAP-23 is a target for calpain cleavage in activated platelets. J. Biol. Chem. 277: 37009-37015.

Sato, K., Saito, Y., and Kawashima, S. 1995. Identification and characterization of membrane-bound calpains in clathrin-coated vesicles from bovine brain. Eur. J. Biochem. 230: 25-31.

Schacher, S. and Proshansky, E. 1983. Neurite regeneration by Aplysia neurons in dissociated cell culture: Modulation by Aplysia hemolymph and the presence of the initial axonal segment. J. Neurosci. 3: 24032413.

Schaffhausen, J.H., Fischer, T.M., and Carew, T.J. 2001. Contribution of postsynaptic $\mathrm{Ca}^{2+}$ to the induction of post-tetanic potentiation in the neural circuit for siphon withdrawal in Aplysia. J. Neurosci. 21: 17391749.

Schikorski, T. and Stevens, C.F. 2001. Morphological correlates of functionally defined synaptic vesicle populations. Nat. Neurosci. 4: 391-395.

Schweizer, F.E. and Ryan, T.A. 2006. The synaptic vesicle: Cycle of exocytosis and endocytosis. Curr. Opin. Neurobiol. 16: 298-304.

Seubert, P., Baudry, M., Dudek, S., and Lynch, G. 1987. Calmodulin stimulates the degradation of brain spectrin by calpain. Synapse 1: 20-24.

Sikorski, A.F. and Goodman, S.R. 1991. The effect of synapsin I phosphorylation upon binding of synaptic vesicles to spectrin. Brain Res. Bull. 27: 195-198.

Sikorski, A.F., Terlecki, G., Zagon, I.S., and Goodman, S.R. 1991. Synapsin Imediated interaction of brain spectrin with synaptic vesicles. J. Cell Biol. 114: $313-318$.

Sikorski, A.F., Sangerman, J., Goodman, S.R., and Critz, S.D. 2000. Spectrin $(\beta S p I I \Sigma 1)$ is an essential component of synaptic transmission. Brain Res. 852: $161-166$.
Siman, R., Baudry, M., and Lynch, G. 1984. Brain fodrin: Substrate for calpain I, an endogenous calcium-activated protease. Proc. Natl. Acad. Sci. 81: 3572-3576.

Sossin, W.S., Diaz-Arrastia, R., and Schwartz, J.H. 1993. Characterization of two isoforms of protein kinase C in the nervous system of Aplysia californica. J. Biol. Chem. 268: 5763-5768.

Stevens, C.F. and Wesseling, J.F. 1998. Activity-dependent modulation of the rate at which synaptic vesicles become available to undergo exocytosis. Neuron 21: 415-424.

Sudhof, T.C. 2004. The synaptic vesicle cycle. Annu. Rev. Neurosci. 27: 509-547.

Sumi, M., Kiuchi, K., Ishikawa, T., Ishii, A., Hagiwara, M., Nagatsu, T., and Hidaka, H. 1991. The newly synthesized selective $\mathrm{Ca}^{2+} /$ calmodulin dependent protein kinase II inhibitor KN-93 reduces dopamine contents in PC12h cells. Biochem. Biophys. Res. Commun. 181: 968-975.

Tsujinaka, T., Kajiwara, Y., Kambayashi, J., Sakon, M., Higuchi, N., Tanaka, T., and Mori, T. 1988. Synthesis of a new cell penetrating calpain inhibitor (calpeptin). Biochem. Biophys. Res. Commun. 153: 12011208.

Wang, L.Y. and Kaczmarek, L.K. 1998. High-frequency firing helps replenish the readily releasable pool of synaptic vesicles. Nature 394: 384-388.

Weis, S., Schneggenburger, R., and Neher, E. 1999. Properties of a model of $\mathrm{Ca}^{++}$-dependent vesicle pool dynamics and short term synaptic depression. Biophys. J. 77: 2418-2429.

Wu, L.G. and Borst, J.G. 1999. The reduced release probability of releasable vesicles during recovery from short-term synaptic depression. Neuron 23: 821-832.

Wu, H.Y. and Lynch, D.R. 2006. Calpain and synaptic function. Mol. Neurobiol. 33: 215-236.

$\mathrm{Xu}$, J., He, L., and Wu, L.G. 2007. Role of $\mathrm{Ca}^{2+}$ channels in short-term synaptic plasticity. Curr. Opin. Neurobiol. 17: 352-359.

Yoshimura, Y., Nomura, T., and Yamauchi, T. 1996. Purification and characterization of active fragment of $\mathrm{Ca}^{2+} /$ calmodulin-dependent protein kinase II from the postsynaptic density in the rat forebrain. $J$. Biochem. 119: 268-273.

Zhao, Y. and Klein, M. 2004. Changes in the readily releasable pool of transmitter and in efficacy of release induced by high-frequency firing at Aplysia sensorimotor synapses in culture. J. Neurophysiol. 91: 15001509.

Zimmer, W.E., Zhao, Y., Sikorski, A.F., Critz, S.D., Sangerman, J., Elferink, L.A., Xu, X.S., and Goodman, S.R. 2000. The domain of brain $\beta$-spectrin responsible for synaptic vesicle association is essential for synaptic transmission. Brain Res. 881: 18-27.

Zimmerman, U.J., Malek, S.K., Liu, L., and Li, H.L. 1999. Proteolysis of synaptobrevin, syntaxin, and SNAP-25 in alveolar epithelial type II cells. IUBMB Life 48: 453-458.

Zucker, R.S. and Regehr, W.G. 2002. Short-term synaptic plasticity. Annu. Rev. Physiol. 64: 355-405.

Received November 5, 2008; accepted in revised form December 10, 2008. 


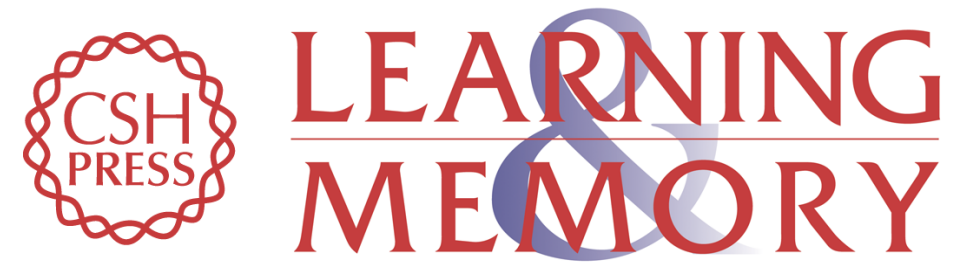

\section{Activity-dependent calpain activation plays a critical role in synaptic facilitation and post-tetanic potentiation}

Arkady Khoutorsky and Micha E. Spira

Learn. Mem. 2009, 16:

Access the most recent version at doi:10.1101//m.1275709

References This article cites 109 articles, 43 of which can be accessed free at:

http://learnmem.cshlp.org/content/16/2/129.full.htmI\#ref-list-1

License

Email Alerting Receive free email alerts when new articles cite this article - sign up in the box at the Service top right corner of the article or click here. 